\title{
DUST AROUND R CORONAE BOREALIS STARS. I. SPITZER/INFRARED SPECTROGRAPH OBSERVATIONS
}

\author{
D. Aníbal García-Hernández ${ }^{1,2}$, N. Kameswara Rao ${ }^{3,4}$, and David L. Lambert ${ }^{4}$ \\ ${ }^{1}$ Instituto de Astrofísica de Canarias, C/ Via Láctea s/n, E-38200 La Laguna, Spain; agarcia@iac.es \\ 2 Departamento de Astrofísica, Universidad de La Laguna (ULL), E-38205 La Laguna, Spain \\ ${ }^{3}$ Indian Institute of Astrophysics, Bangalore 560 034, India; nkrao@iiap.res.in \\ ${ }^{4}$ W. J. McDonald Observatory, The University of Texas at Austin, 1 University Station, \\ C1400. Austin, TX 78712-0259, USA; d11@astro.as.utexas.edu \\ Received 2011 May 10; accepted 2011 July 6; published 2011 September 2
}

\begin{abstract}
Spitzer/infrared spectrograph (IRS) spectra from 5 to $37 \mu \mathrm{m}$ for a complete sample of $31 \mathrm{R}$ Coronae Borealis stars (RCBs) are presented. These spectra are combined with optical and near-infrared photometry of each RCB at maximum light to compile a spectral energy distribution (SED). The SEDs are fitted with blackbody flux distributions and estimates are made of the ratio of the infrared flux from circumstellar dust to the flux emitted by the star. Comparisons for 29 of the 31 stars are made with the Infrared Astronomical Satellite (IRAS) fluxes from three decades earlier: Spitzer and IRAS fluxes at $12 \mu \mathrm{m}$ and $25 \mu \mathrm{m}$ are essentially equal for all but a minority of the sample. For this minority, the IRAS to Spitzer flux ratio exceeds a factor of three. The outliers are suggested to be stars where formation of a dust cloud or dust puff is a rare event. A single puff ejected prior to the IRAS observations may have been reobserved by Spitzer as a cooler puff at a greater distance from the RCB. RCBs which experience more frequent optical declines have, in general, a circumstellar environment containing puffs subtending a larger solid angle at the star and a quasi-constant infrared flux. Yet, the estimated subtended solid angles and the blackbody temperatures of the dust show a systematic evolution to lower solid angles and cooler temperatures in the interval between IRAS and Spitzer. Dust emission by these RCBs and those in the LMC is similar in terms of total $24 \mu \mathrm{m}$ luminosity and [8.0]-[24.0] color index.
\end{abstract}

Key words: circumstellar matter - dust, extinction - infrared: stars - stars: chemically peculiar - white dwarfs

\section{INTRODUCTION}

The R Coronae Borealis (here, $\mathrm{RCB}$ ) stars are notable for two distinct peculiarities (Clayton 1996). First, they are hydrogenpoor, helium-rich supergiants: the $\mathrm{H}$ deficiencies range from about 10 to 100 to at least $10^{8}$. Second, the RCB stars experience unpredictable and rapid declines in brightness: declines of 2-8 mag in the visual occurring at intervals of less than a year to greater than 20 years last from weeks to months to years. These declines are caused by formation of a cloud of carbon soot above the Earth-facing surface of the star. Discovery of an infrared excess confirmed the obvious suspicion that the stars were dust producers (Stein et al. 1969; Feast et al. 1997). Typical blackbody temperatures of the dust run from about $400 \mathrm{~K}$ to $900 \mathrm{~K}$. In a representative case, about one-third of the photospheric flux is absorbed by dust and reemitted in the infrared. The observation that the infrared flux may be little affected by a decline shows that the dust is distributed in clouds around the star (Forrest et al. 1972). Recently, high angular resolution images of $\mathrm{RY} \mathrm{Sgr}$, a bright $\mathrm{RCB}$, at 2.2, 4.5, and $8-13 \mu \mathrm{m}$ showed clearly that the dust is indeed distributed in clouds (de Laverny \& Mékarnia 2004; Leão et al. 2007; Bright et al. 2011). Infrared Astronomical Satellite (IRAS) photometry at long wavelengths showed that, in addition to the warm dust, some RCBs have dust at a lower temperature (say, 30-100 K) and, therefore, at large distances from the star (Rao \& Nandy 1986; Walker 1986; Gillett et al. 1986).

Each of the two principal peculiarities prompts leading questions. In the case of the $\mathrm{H}$ deficiency, that question is- what are the evolutionary origins of the RCBs that result in an $\mathrm{H}$-poor stars? Two scenarios remain under active consideration for the RCBs and their putative relatives the $\mathrm{H}$-deficient carbon ( $\mathrm{HdC}$ ) stars to lower temperatures and the extreme helium stars to higher temperature. In one, the $\mathrm{H}$-poor supergiant is formed from the merger of an $\mathrm{He}$ white dwarf with a $\mathrm{C}-\mathrm{O}$ white dwarf; the double-degenerate (DD) scenario. In the competing picture, the $\mathrm{H}$-poor supergiant results from a final post-asymptotic giant branch (AGB) shell flash in the central star of a planetary nebula; the so-called final-flash (FF) scenario. In both cases, the trigger - the merger or the FF-transforms a white dwarf into an $\mathrm{H}$-poor supergiant for a period of a few thousand years. There is evidence that both the DD and FF scenarios occur but the DD scenario seems likely to account for the majority of the RCBs.

Several insights into the origins of the RCBs are coming from spectroscopic determinations of the stellar chemical compositions (Lambert \& Rao 1994; Asplund et al. 2000; Clayton et al. 2005, 2007; García-Hernández et al. 2009a, 2010a; Jeffery et al. 2011; Pandey \& Lambert 2011). Detailed abundance analyses, which are possible for the warm RCBs but rarely undertaken for the cool RCBs with their spectra rich in molecular lines, suggest that many RCBs are likely fruits of the DD scenario. A few RCBs show several highly unusual abundance signatures and, in particular, very distinctive $\mathrm{Si} / \mathrm{Fe}$ and $\mathrm{S} / \mathrm{Fe}$ ratios. Such stars are called "minority" RCBs—see Lambert \& Rao (1994) who introduced the terms "majority RCB" and "minority RCB" star. A rare class of hot RCBs is discussed by De Marco et al. (2002) and includes DY Cen, a minority RCB.

The other principal peculiarity-the unpredictable declines - stimulates a series of questions about the dust around RCB stars such as: What is the composition of the dust? Where does dust form relative to the stellar surface? What triggers formation of the obscuring cloud? How frequently do dust clouds form? Does dust form at preferred latitudes on the star or are formation sites spread uniformly across the stellar surface? Clayton (1996) reviews evidence pertinent to these questions. Perhaps 
the key novel theoretical idea of recent times comes from Woitke et al. (1996) who developed a model in which a pulsationinduced shock triggers dust nucleation near the star (one to two stellar radii out) as gas behind the outward propagating shock cools below the condensation temperature (say, $<1500 \mathrm{~K}$ ) in the star's upper atmosphere. The presence of cool gas during light minima has now been detected in three RCBs: R CrB (Rao et al. 2006; Rao \& Lambert 2010), V854 Cen (Rao \& Lambert 2000), and V CrA (Rao \& Lambert 2008). Light variations at maximum light are common among RCBs and are generally interpreted as arising from pulsations. Absorption line splitting suggestive of an atmospheric shock is regularly observed for RY Sgr (Danziger 1965; Cottrell \& Lambert 1982) and occasionally for R CrB. Pugach (1977) noted a correlation between the onset of a light decline for RY Sgr and the pulsation phase. Crause et al. (2007) have shown from long-term photometric studies of four RCBs that a decline occurs at a particular phase of the pulsation cycle, although not every pulsation cycle results in a decline. Thus, evidence is accumulating that dust formation occurs near the star. Radiation pressure on the dust grains is considered to drive them rapidly outward.

Clues to several of the questions concerning the dust are contained in the shape of the infrared continuum emitted by the circumstellar dust, the presence of emission or absorption features imposed on that continuum and on the temporal variation of the infrared flux. Ground-based infrared spectrophotometry has revealed a smooth continuum in the atmospheric windows; strong emission and absorption features have not been seen. Inability to observe in regions blocked by Earth's atmosphere is an especially serious problem in searching for features attributable to dust. Infrared Space Observatory (ISO) spectra obtained for just three (the brightest) RCBs-R CrB, RY Sgr, and V854 Cen-at a resolution of $R=1000$ showed for the first time some excess emission over a quasi-blackbody continuum (Lambert et al. 2001). Broad unidentified emission features were seen centered on about $6 \mu \mathrm{m}$ and $13 \mu \mathrm{m}$. Emission features from $4 \mu \mathrm{m}$ to $15 \mu \mathrm{m}$ for V854 Cen but not for R CrB and RY Sgr showed a resemblance to a laboratory spectrum of hydrogenated amorphous carbon (Colangeli et al. 1995; Scott et al. 1997).

With the advent of the Spitzer Space Telescope, it became possible for the first time to extend low-resolution infrared spectroscopy to a much larger sample of RCBs. In this paper, we present a library of infrared Spitzer/IRS spectra for a large sample (31) of RCB stars. Spectra are characterized by a fit of blackbodies to optical and infrared photometry and the Spitzer spectra. Estimates are provided of the infrared flux emitted by dust to the total stellar flux. Comparisons are made with previously reported measurements of the infrared flux, principally the $12 \mu \mathrm{m}$ and $25 \mu \mathrm{m}$ flux measurements from IRAS. Infrared emission features superimposed on the blackbody continuua will be discussed in detail in a subsequent paper. Emission spectra of DY Cen and V854 Cen showing features from polycyclic aromatic hydrocarbons (PAHs) and $\mathrm{C}_{60}$ have been discussed by García-Hernández et al. (2011). Spitzer/ IRS spectra of the hot RCB stars V348 Sgr and HV 2671 are presented in Clayton et al. (2011).

Section 2 describes our sample of RCB stars, the Spitzer/IRS and some ground-based photometric observations obtained at about the same time as the Spitzer observations, and the IRAS observations. Section 3 constructs the spectral energy distributions (SEDs) from $\sim 0.4$ to $38 \mu \mathrm{m}$ from optical and infrared observations, where the SEDs are fitted using blackbodies for the star and the dust for each object. Spitzer and IRAS observations are compared and discussed in Section 4, while a comparison of the RCB dust emission in different metallicity environments is offered in Section 5. Finally, the paper concludes with Section 6.

\section{THE SAMPLE AND OBSERVATIONS}

\subsection{The RCB Sample}

Our main goal in obtaining Spitzer observations was to compile a library of infrared spectra for as complete a sample of RCBs as possible. Table 1 lists the 31 RCB stars included in this study together with some relevant information such as coordinates, the date of the Spitzer observation, Spitzer program ID, etc. Eighteen RCBs were in our approved GO program. An additional 13 warm RCBs were found in the public Spitzer database. The sample provides comprehensive coverage of the hot RCBs, warm RCBs, and includes several of the coolest RCBs. The target list in Table 1 is also identified according to the categories A, B, or C. Category A corresponds to warm RCBs across the composition range (13 stars) where compositions are taken from Asplund et al. (2000). Category B is assigned to the few (5) minority RCB stars. The coolest RCB stars belong to category C (13 stars). Note that minority stars V3795 Sgr, VZ Sgr, V CrA, V854 Cen, and DY Cen fall in categories A and $\mathrm{B}(\mathrm{AB})$ and $\mathrm{Z}$ UMi is assigned to the category BC. The RCB star HV 2671 in the Large Magellanic Cloud (LMC) has yet to be assigned to one of the categories; De Marco et al. (2002) note that HV 2671 and V348 Sgr have almost identical optical spectra but the Spitzer spectra are very different although HV 2671 shows similarities with V854 Cen (Clayton et al. 2011). Also in Table 1, we indicate whether a star was observed at or below maximum light.

\subsection{Spitzer Observations}

The infrared spectra were taken with the Infrared Spectrograph (IRS; Houck et al. 2004) on board the Spitzer Space Telescope (Werner et al. 2004). We obtained 5.2-37.2 $\mu \mathrm{m}$ spectra for 18 sources in our sample under our General Observer Program (\#50212, PI: D. L. Lambert) that was carried out between 2008 April and October. We used a combination of IRS short-low (5.2-14.5 $\mu \mathrm{m} ; 64<R<128$, here SL), short-high (9.9-19.6 $\mu \mathrm{m}$, here $\mathrm{SH})$, and long-high (18.7-37.2 $\mu \mathrm{m}$, here LH) observations $(R \sim 600)$. Since IRAS fluxes at 12 and $25 \mu \mathrm{m}$ are available for all sources in our sample, we assumed we had a priori knowledge of the mean brightness of each source at the different wavelengths covered by IRS. Most of these sources are very bright (with mid-IR SEDs peaking at $\sim 12 \mu \mathrm{m}$ ), and two cycles of $6 \mathrm{~s}$ in each of the three modules were used. For those sources with lower flux densities at $25 \mu \mathrm{m}$, four cycles of $14 \mathrm{~s}$ were employed in the LH module. We typically reached a signal-to-noise ratio $(\mathrm{S} / \mathrm{N})$ larger than 50 in the $\mathrm{SL}$ and $\mathrm{SH}$ modules; these modules cover the $5.2-19.5 \mu \mathrm{m}$ range where most of the spectral features of our interest fall. However, the $\mathrm{S} / \mathrm{N}$ achieved is generally lower in the LH module. For three stars (those sources brighter than $5.5 \mathrm{Jy}$ at $12 \mu \mathrm{m}$; see Table 1) we did not obtain spectroscopy in the SL module in order to avoid saturation.

IRS spectra for 13 other stars were retrieved from the Spitzer database. These spectra were taken by different observers and using different module combinations (see Table 1). In general, the quality of these spectra is also very $\operatorname{good}(\mathrm{S} / \mathrm{N} \geqslant 50)$; especially when the SL and LL (long-low: 14.0-38.0 $\mu \mathrm{m}$; 
Table 1

The RCB Stars Sample ${ }^{a}$

\begin{tabular}{|c|c|c|c|c|c|c|c|c|c|}
\hline RCB Star & R.A.J2000 & Decl.J2000 & Category & $\begin{array}{l}F_{12} \\
(\mathrm{Jy})\end{array}$ & $\begin{array}{l}F_{25} \\
(\mathrm{Jy})\end{array}$ & $\begin{array}{c}\text { Obs. Date } \\
\text { (yyyy/mm/dd) }\end{array}$ & Var. $^{\mathrm{b}}$ & Modules & Program \\
\hline UV Cas & $23: 02: 14.62$ & $+59: 36: 36.6$ & A & 3.81 & 1.28 & $2008 / 08 / 12$ & $\max$ & SL,SH,LH & 50212 \\
\hline S Aps & $15: 09: 24.53$ & $-72: 03: 45.1$ & $\mathrm{C}$ & 2.71 & 1.02 & $2008 / 04 / 25$ & $\max$ & SL,SH,LH & 50212 \\
\hline SV Sge & 19:08:11.76 & $+17: 37: 41.2$ & $\mathrm{C}$ & 3.29 & 1.66 & $2008 / 05 / 26$ & $\max$ & SL,SH,LH & 50212 \\
\hline Z UMi & $15: 02: 01.33$ & $+83: 03: 48.6$ & $\mathrm{C}$ & 2.12 & 0.82 & $2008 / 10 / 20$ & $\min [2]$ & SL,SH,LH & 50212 \\
\hline V1783 Sgr & $18: 04: 49.74$ & $-32: 43: 13.4$ & $\mathrm{C}$ & 3.20 & 1.26 & $2008 / 04 / 25$ & $\max$ & SL,SH,LH & 50212 \\
\hline WX CrA & $18: 08: 50.48$ & $-37: 19: 43.2$ & $\mathrm{C}$ & 2.31 & 0.77 & $2008 / 10 / 10$ & $\max$ & SL,SH,LH & 50212 \\
\hline V3795 Sgr & $18: 13: 23.58$ & $-25: 46: 40.8$ & $\mathrm{AB}$ & 4.17 & 1.80 & $2008 / 04 / 25$ & $\max$ & SL,SH,LH & 50212 \\
\hline V1157 Sgr & $19: 10: 11.83$ & $-20: 29: 42.1$ & $\mathrm{C}$ & 3.21 & 1.17 & $2008 / 06 / 02$ & $\min [2]$ & SL,SH,LH & 50212 \\
\hline Y Mus & $13: 05: 48.19$ & $-65: 30: 46.7$ & A & 1.02 & 0.35 & $2008 / 04 / 25$ & $\max$ & SL,SH,LH & 50212 \\
\hline V739 Sgr & $18: 13: 10.54$ & $-30: 16: 14.7$ & $\mathrm{C}$ & 1.27 & 0.36 & $2008 / 04 / 25$ & $\max$ & SL,SH,LH & 50212 \\
\hline VZ Sgr & 18:15:08.58 & $-29: 42: 29.4$ & $\mathrm{AB}$ & 1.11 & 0.59 & $2008 / 04 / 25$ & $\min [4]$ & SL,SH,LH & 50212 \\
\hline U Aqr & $22: 03: 19.70$ & $-16: 37: 35.2$ & $\mathrm{C}$ & 1.11 & 0.51 & $2008 / 06 / 29$ & $\max$ & SL,SH,LH & 50212 \\
\hline MACHOJ181933 & $18: 19: 33.75$ & $-28: 35: 58.0$ & $\mathrm{C}$ & $\ldots$ & $\ldots$ & $2008 / 04 / 25$ & $\max$ & SL,SH,LH & 50212 \\
\hline ES Aql & $19: 32: 21.61$ & $-00: 11: 31.0$ & $\mathrm{C}$ & 1.43 & 0.52 & $2008 / 05 / 27$ & $\max$ & SL,SH,LH & 50212 \\
\hline FH Sct & $18: 45: 14.84$ & $-09: 25: 36.1$ & A & 0.62 & 0.49 & $2008 / 06 / 02$ & $\max$ & SL,SH,LH & 50212 \\
\hline SU Tau & $05: 49: 03.73$ & $+19: 04: 22.0$ & A & 9.50 & 4.14 & $2008 / 04 / 28$ & $\max$ & SH,LH & 50212 \\
\hline DY Per & $02: 35: 17.13$ & $+56: 08: 44.7$ & $\mathrm{C}$ & 8.65 & 1.71 & $2008 / 09 / 13$ & $\max$ & SH,LH & 50212 \\
\hline V517 Oph & $17: 15: 19.74$ & $-29: 05: 37.6$ & $\mathrm{C}$ & 7.81 & 2.53 & $2008 / 04 / 25$ & $\min [2.4]$ & SH,LH & 50212 \\
\hline $\mathrm{V} \mathrm{CrA}$ & $18: 47: 32.30$ & $-38: 09: 32.3$ & $\mathrm{AB}$ & 5.66 & 2.46 & $2005 / 09 / 14$ & $\max$ & SL,LL & 7 \\
\hline RZ Nor & $16: 32: 41.66$ & $-53: 15: 33.2$ & A & 3.45 & 1.75 & $2006 / 03 / 22$ & $\max$ & SL,LL & 7 \\
\hline RT Nor & $16: 24: 18.68$ & $-59: 20: 38.6$ & A & 0.93 & 0.40 & $2005 / 04 / 21$ & $\max$ & SL,LL & 7 \\
\hline RS Tel & $18: 18: 51.22$ & $-46: 32: 53.4$ & A & 1.54 & 0.71 & $2005 / 09 / 10$ & $\max$ & SL,LL & 7 \\
\hline V482 Cyg & $19: 59: 42.57$ & $+33: 59: 27.9$ & A & 0.98 & 0.41 & $2004 / 11 / 14$ & $\max$ & SL,LL & 7 \\
\hline MV Sgr & $18: 44: 31.97$ & $-20: 57: 12.8$ & A & 0.60 & 1.57 & $2005 / 04 / 18$ & $\max$ & SL,SH,LH & 3362 \\
\hline RY Sgr & $19: 16: 32.76$ & $-33: 31: 20.4$ & A & 77.20 & 26.20 & $2004 / 10 / 21$ & $\min [2.6]$ & SH,LH & 3362 \\
\hline V854 Cen & $14: 34: 49.41$ & $-39: 33: 19.2$ & $\mathrm{AB}$ & 23.00 & 7.82 & $2007 / 09 / 07$ & $\min [5.5]$ & SL,SH,LH & 30077 \\
\hline UW Cen & $12: 43: 17.18$ & $-54: 31: 40.7$ & A & 7.85 & 5.75 & $2008 / 08 / 17$ & $\max$ & SL,SH,LL & 40061 \\
\hline DY Cen & $13: 25: 34.08$ & $-54: 14: 43.1$ & $\mathrm{AB}$ & 0.91 & 0.93 & $2008 / 08 / 17$ & $\max$ & SL,LL & 40061 \\
\hline $\mathrm{R} \mathrm{CrB}$ & $15: 48: 34.41$ & $+28: 09: 24.3$ & A & 17.10 & 3.94 & $2004 / 07 / 17$ & $\max$ & $\mathrm{SH}$ & 93 \\
\hline V348 Sgr & $18: 40: 19.93$ & $-22: 54: 29.3$ & A & 5.53 & 3.00 & $2006 / 10 / 22$ & $\min [2.7]$ & SL,LL & 30380 \\
\hline HV 2671 & $05: 3348.94$ & $-70: 13: 23.4$ & LMC-RCB & $\ldots$ & $\ldots$ & $2006 / 11 / 14$ & $\ldots$ & SL,LL & 30380 \\
\hline
\end{tabular}

Notes.

a The first 18 RCB stars were observed with Spitzer by us (Program 50212), while the rest of the stars were observed by other programs and the data were retrieved from the Spitzer database (see the text).

b Variability status during the Spitzer observations; max: the star was observed at (or slightly below; e.g., <0.6-0.8 mag in $V$ ) maximum light. min: the star was observed during minimum light and the number in brackets indicate the $V$ magnitudes below maximum.

$64<R<128$ ) modules are used. The Spitzer/IRS spectra of the infrared-bright RCB stars V854 Cen, RY Sgr, and $\mathrm{R} \mathrm{CrB}$ - previously observed by the Infrared Space Observatory (ISO) satellite (Lambert et al. 2001) —are included in this subgroup.

We retrieved the one-dimensional infrared spectra processed by the Spitzer data reduction pipeline (versions 15.3.0, 16.1.0, 17.2.0, 18.0.2, and 18.7.0) for all sources in our sample from the Spitzer database. These post-BCD (Basic Calibrated Data) products (one spectrum for each nod position) are automatically reduced by the IRS Custom Extractor (SPICE) with a pointsource aperture. The automatic data reduction includes the extraction from the two-dimensional (2D) images as well as the wavelength and flux calibration. It is to be noted here that for the SL and LL data, the two nod position 2D images are subtracted in order to cancel out the sky background. However, for the high-resolution modules no background subtraction is done since no sky measurements were taken; the SH and LH slits are too small for on-slit background subtraction. The Spitzercontributed software SMART (Higdon et al. 2004) was later used for cleaning of residual bad pixels, spurious jumps and glitches, and for smoothing and merging into one final spectrum per source. Note that all sources in our sample are bright pointlike objects for which the automatic data reduction pipeline works very well; no significant differences are found between these spectra and those reduced manually.

We found a good match (i.e., better than 5\%) between the different modules for approximately half of the sample stars. Most of the rest of the stars displayed a very good match between the SL and SH modules, confirming their point-like nature, but the LH data showed a flux excess of about $5 \%-20 \%$. We attribute this mismatch to the fact that these $\mathrm{LH}$ fluxes are more uncertain and to possible background emission. Indeed, several of these stars are located toward high extinction lines of sight (i.e., their infrared spectra are affected by amorphous silicate absorptions from the diffuse interstellar medium; see below). Thus, we applied a correction factor to the LH observations in order to scale them to the SH spectra. It should be noted that only the RCB star VZ Sgr seems to be slightly extended at infrared wavelengths.

Reduced spectra- $\lambda F_{\lambda}$ versus $\lambda$-are shown in Figures 1-5 for the complete sample of 31 stars.

\subsection{Ground-based Optical/Near-IR Photometry}

To complement the Spitzer observations, we carried out photometric observations in the optical and near-infrared for some of the sample. Our intention was to ascertain a star's status (i.e., if the stars were at maximum or minimum light) 


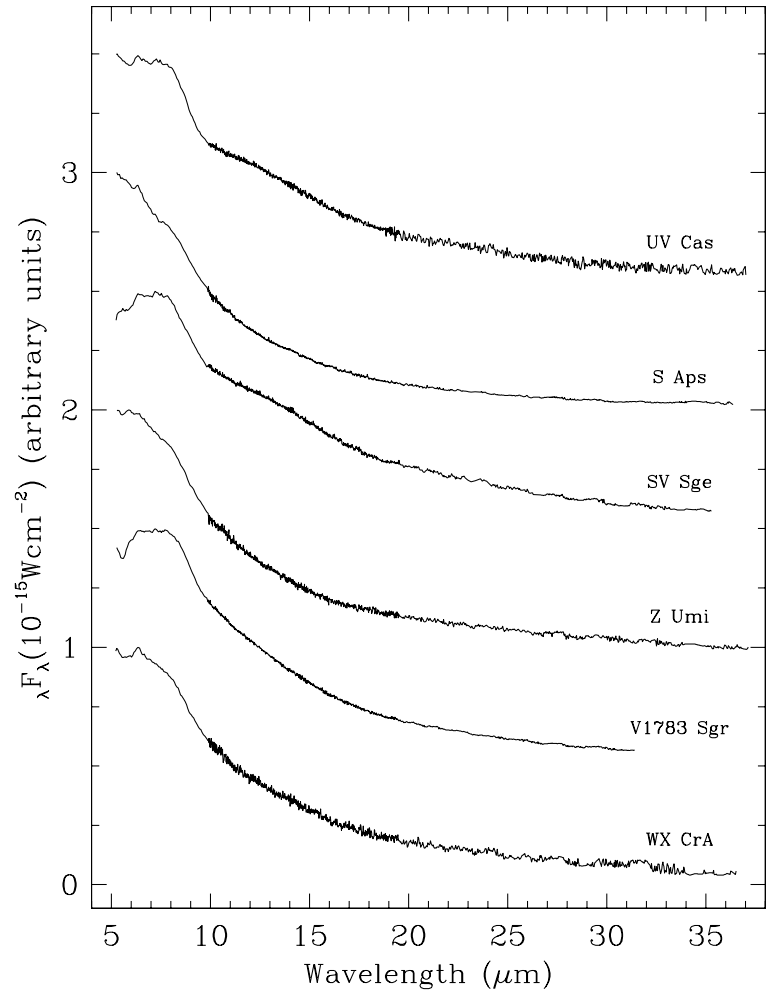

Figure 1. Spitzer /IRS reduced spectra over the full wavelength range $\sim 5-37 \mu \mathrm{m}$ for (from top to bottom) UV Cas, S Aps, SV Sge, Z UMi, V1783 Sgr, and WX CrA. Note that the spectra are normalized and displaced for clarity.

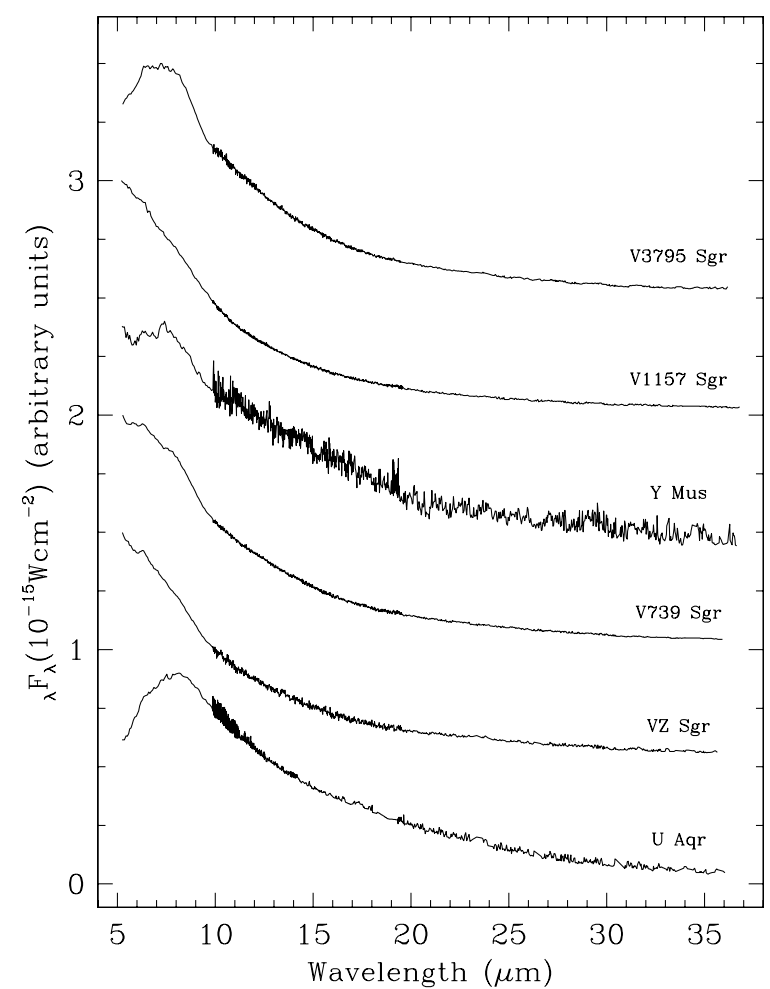

Figure 2. Spitzer/IRS reduced spectra over the full wavelength range $\sim 5-37 \mu \mathrm{m}$ for V3795 Sgr, V1157 Sgr, Y Mus, V739 Sgr, VZ Sgr, and U Aqr. Note that the spectra are normalized and displaced for clarity.

during the Spitzer observations. However, these "simultaneous" photometric data were obtained only for the $18 \mathrm{RCB}$ stars observed with Spitzer through Program 50212 (Table 2). If a star

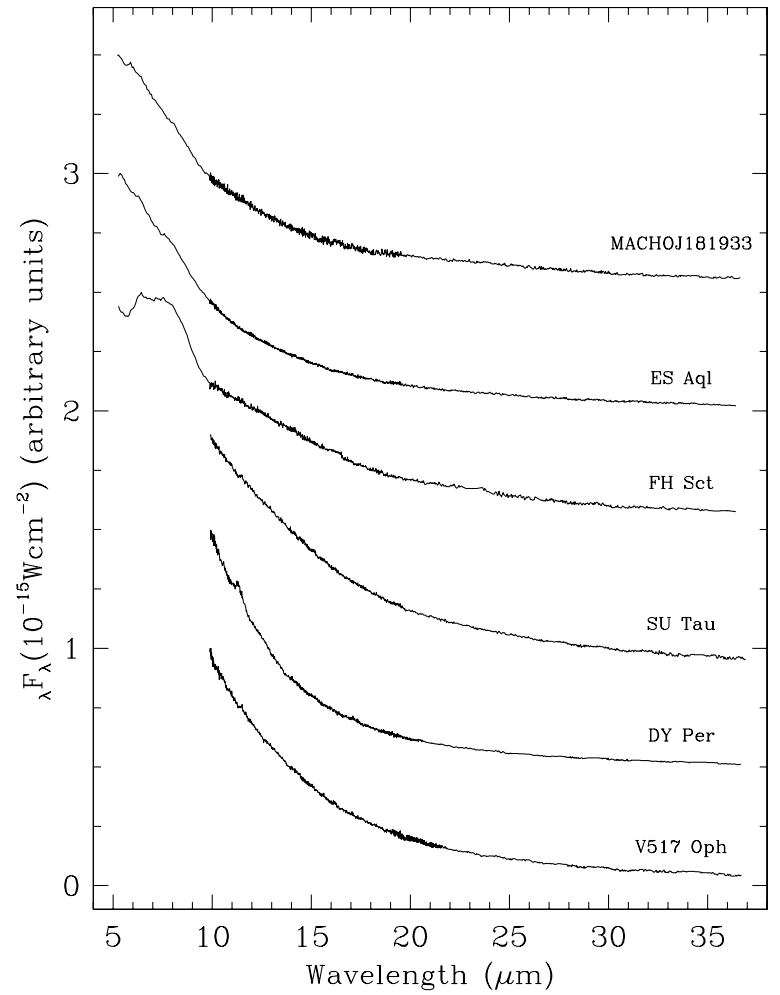

Figure 3. Spitzer/IRS reduced spectra over the full wavelength range $\sim 5-37 \mu \mathrm{m}$ for MACHOJ181933, ES Aql, FH Sct, SU Tau, DY Per, and V517 Oph. Note that the spectra are normalized and displaced for clarity.

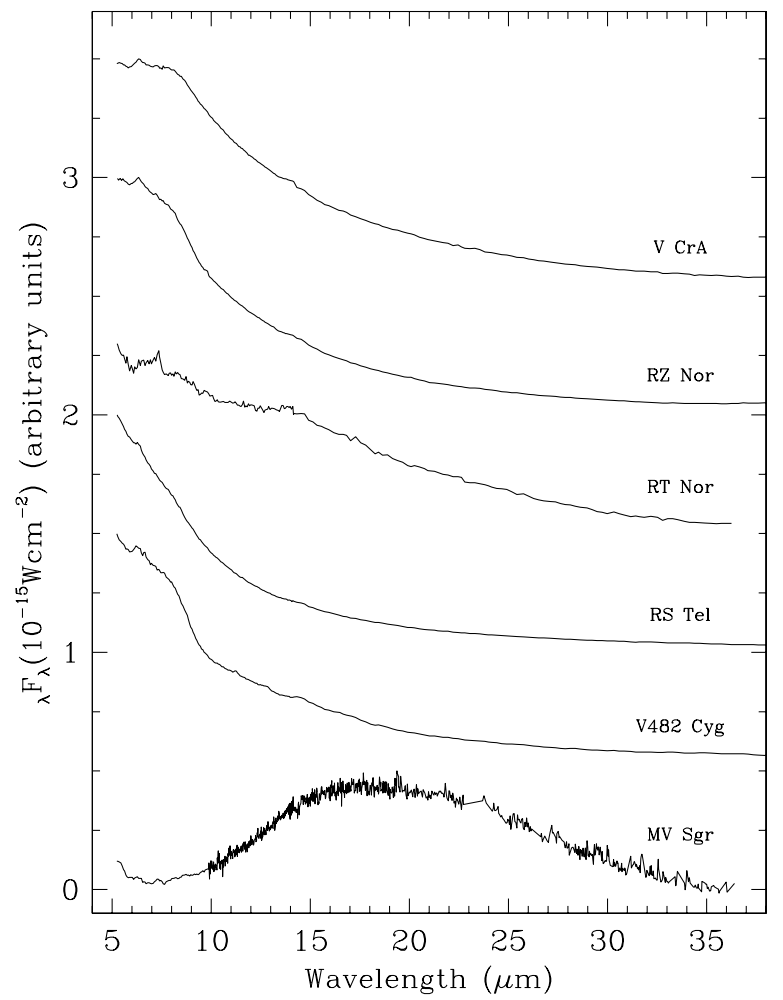

Figure 4. Spitzer/IRS reduced spectra over the full wavelength range $\sim 5-37 \mu \mathrm{m}$ for V CrA, RZ Nor, RT Nor, RS Tel, V482 Cyg, and MV Sgr. Note that the spectra are normalized and displaced for clarity.

was at maximum light, the photometry is used in the construction of the SEDs from the visible to $\sim 40 \mu \mathrm{m}$ in our sample stars. For stars not at maximum light, photometry from the literature 


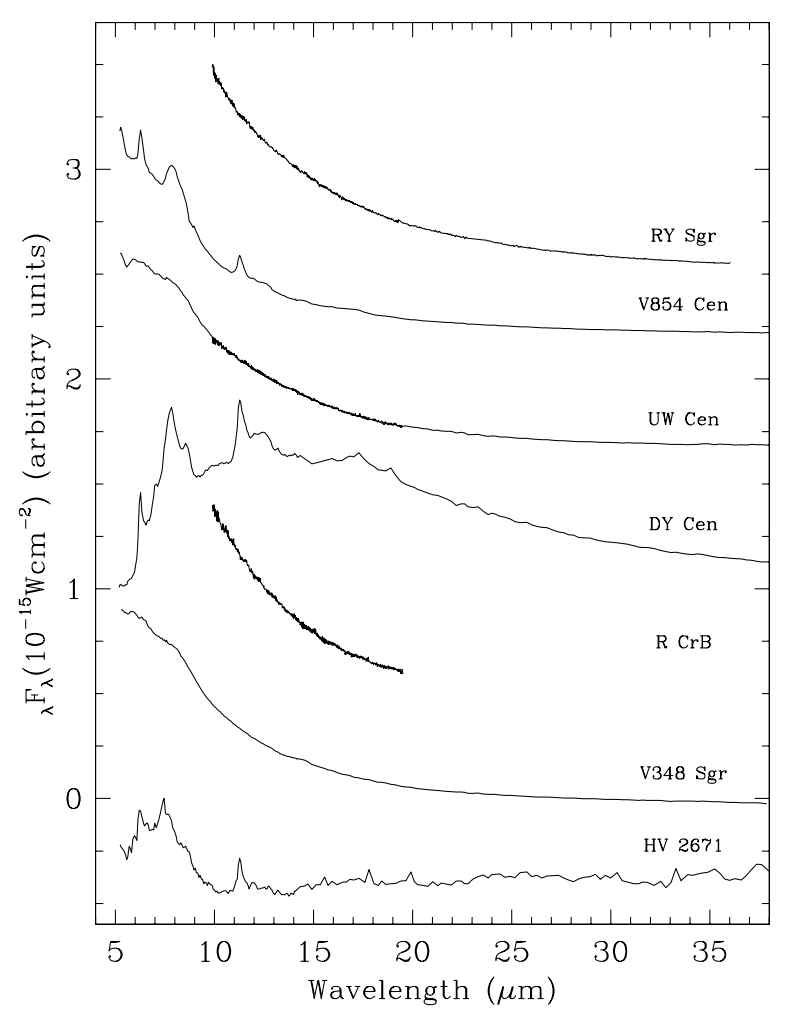

Figure 5. Spitzer/IRS reduced spectra over the full wavelength range $\sim 5-37 \mu \mathrm{m}$ for RY Sgr, V854 Cen, UW Cen, DY Cen, R CrB, V348 Sgr, and HV 2671. Note that the spectra are normalized and displaced for clarity.

was used to establish the stellar energy distribution at maximum light across the optical.

Optical photometry in the Johnson-Bessell $V, R$, and $I$ filters was obtained with the IAC-80 telescope (Observatorio del Teide, Spain) equipped with the CAMELOT $\mathrm{CCD}^{5}$ for more details. The observations were done as near as possible to the Spitzer observation dates and sometimes the stars were observed twice (i.e., before and after Spitzer). The VRI magnitudes for each star were derived by using standard aperture photometry tasks in IRAF. ${ }^{6}$ The flux calibration was done by using the photometric calibration for $\mathrm{CAMELOT}^{7}$ and making use of standard stars observed on the same night. The use of this average photometric calibration implies that our derived VRI magnitudes are precise to $\sim 0.15 \mathrm{mag}$. This error in the optical magnitudes is more than enough for our purposes, that is, to know the variability status of these RCB stars. Table 2 displays a log of the optical observations done (e.g., the observation dates) together with the $V R I$ photometry for each star observed.

$J H K L$ photometry (Table 2) was obtained at the South African Astrophysical Observatory (SAAO) with the $0.75 \mathrm{~m}$ telescope by F. Van Wyk at our request. These observations are on the SAAO system using Carter (1990) standards.

\footnotetext{
5 See e.g., http://www.iac.es/telescopes/pages/es/inicio/ instrumentos/camelot.php

6 Image Reduction and Analysis Facility (IRAF) software is distributed by the National Optical Astronomy Observatory, which is operated by the

Association of Universities for Research in Astronomy, Inc., under cooperative agreement with the National Science Foundation.

7 See http://www.iac.es/telescopes/pages/en/home/utilities.php \#camelot-calibration for the average extinction coefficients, color terms, etc.
}

\section{SPECTRAL ENERGY DISTRIBUTIONS}

\subsection{Methodology}

In this section, we present the SED from $\sim 0.4$ to $40 \mu \mathrm{m}$ for each RCB star. UBVRIJHKLMN magnitudes are converted to fluxes with the magnitude-flux and effective wavelength calibrations taken from Tokunaga (2000). For a majority of the stars, there are $U B V R I J H K$ magnitudes in the literature. Some data are available for $L$ magnitudes and a few for $M N$ magnitudes. In addition, there are the ground-based measurements in Table 2.

The goal was to construct the "stellar" SED from observations made when the star was at maximum light. This SED uses the $U B V R I J H K$ fluxes except that for a few stars some observations of $K$ and possibly $H$ are contaminated by emission by dust. In addition to the Spitzer spectrum of the dust emission, we consider the IRAS $12 \mu \mathrm{m}$ and $25 \mu \mathrm{m}$ measurements and available $L M N$ photometry. The $L M N$, Spitzer, and IRAS fluxes are primarily from the circumstellar dust. As is well known, optical and infrared variability are not tightly coupled-see below and especially Feast et al. (1997).

These SEDs are corrected for interstellar reddening provided by the line of sight to the RCB. Spitzer spectra require a correction for absorptions at $9.7 \mu \mathrm{m}$ and $18 \mu \mathrm{m}$, attributable to interstellar silicates. For this correction, the reddening curve is adopted from Chiar \& Tielens (2006) by taking $A(K) / A(V)=$ 0.114 (Cardelli et al. 1989) with an extrapolation from 27 to $38 \mu \mathrm{m}$ assuming the same slope as between 23 and $27 \mu \mathrm{m}$. The correction was generally ignored for stars where the predicted reddening was less than about $E(B-V)$ of 0.4 . The correction for the $9.7 \mu \mathrm{m}$ interstellar absorption can have a particularly strong effect on the profile and intensity of the $6-10 \mu \mathrm{m}$ emission feature, the subject of a subsequent paper.

A few stars in Table 1 were observed by Spitzer in decline. For these stars, we assemble the stellar SEDs from published photometry at maximum light; we do not use the contemporary photometry, if available, in Table 2. This is then combined with the Spitzer spectrum to provide that star's SED which is corrected, as usual, for interstellar reddening.

In the case of the IRAS $12 \mu \mathrm{m}$ and $25 \mu \mathrm{m}$ photometry, the measurements were color corrected. Color-corrected flux densities were mostly obtained from Walker (1986). In some cases, measurements in the IRAS point-source catalog were corrected following the prescriptions given by Beichman et al. (1988 - Table VI.C.6).

Each reddening-corrected SED was fitted with a combination of blackbodies with one blackbody at the stellar effective temperature and one or two blackbodies to represent the infrared circumstellar component. In a few cases, a third circumstellar blackbody was considered. Table 3 summarizes the fits by giving the temperature and the estimated flux ratio for the blackbody relative to the stellar flux where $R=f_{\text {cool }} / f_{\text {star }}$ is referred to as the covering factor. The sum of the $R$-values for a given star is essentially independent of the assumption that dust emission may be represented by one or more blackbodies. Our $R$-values do not include the small contribution from the 6-10 $\mu \mathrm{m}$ emission feature. Entries are given for both the Spitzer and IRAS fits except where there is no significant difference between the two fits.

All sources of photometry and interstellar reddening are identified below where brief descriptions are also given of other characteristics of each star (frequency of declines, comparison with IRAS and other IR fluxes, etc.). A primary source on 
Table 2

Optical and Near-infrared Photometry ${ }^{\mathrm{a}}$

\begin{tabular}{|c|c|c|c|c|c|}
\hline RCB Star & $\begin{array}{l}\text { Spitzer Obs. Date } \\
\text { (yyyy/mm/dd) }\end{array}$ & $\begin{array}{l}\text { IAC-80 Obs. Date } \\
(\text { yyyy/mm/dd) }\end{array}$ & $V / R / I^{\mathrm{b}}$ & $\begin{array}{l}\text { SAAO Obs. Date } \\
(\text { yyyy } / \mathrm{mm} / \mathrm{dd})\end{array}$ & $J / H / K / L$ \\
\hline UV Cas & $2008 / 08 / 12$ & $\begin{array}{l}2008 / 08 / 11 \\
2008 / 08 / 13\end{array}$ & $\begin{array}{l}10.73 / 9.86 / 9.01 \\
10.72 / 9.86 / 8.99\end{array}$ & $\ldots$ & $\ldots$ \\
\hline S Aps & $2008 / 04 / 25$ & $\ldots$ & $\ldots$ & $2008 / 04 / 24$ & $7.78 / 7.16 / 6.67 / 5.31$ \\
\hline SV Sge & $2008 / 05 / 26$ & $2008 / 05 / 30$ & $10.68 / 9.60 / 8.50$ & $\ldots$ & $\ldots$ \\
\hline Z UMi & $2008 / 10 / 20$ & $\ldots$ & $\ldots$ & & \\
\hline V1783 Sgr & $2008 / 04 / 25$ & $\begin{array}{l}2008 / 04 / 24 \\
2008 / 05 / 04\end{array}$ & $\begin{array}{l}10.60 / 9.71 / 8.86 \\
10.71 / 9.82 / 8.93\end{array}$ & $2008 / 04 / 29$ & $8.74 / 8.34 / 7.96 / \cdots$ \\
\hline WX CrA & $2008 / 10 / 10$ & $\ldots$ & $\ldots$ & & \\
\hline V3795 Sgr & $2008 / 04 / 25$ & $\begin{array}{l}2008 / 04 / 24 \\
2008 / 05 / 04\end{array}$ & $\begin{array}{l}11.57 / 10.94 / 10.27 \\
11.57 / 10.94 / 10.29\end{array}$ & $2008 / 04 / 29$ & $9.14 / 8.63 / 8.24 / \cdots$ \\
\hline $\begin{array}{l}\text { V1157 Sgr } \\
\text { Y Mus }\end{array}$ & $\begin{array}{l}2008 / 06 / 02 \\
2008 / 04 / 25\end{array}$ & $\begin{array}{c}2008 / 06 / 03 \\
\ldots\end{array}$ & $\begin{array}{c}13.20 / 12.23 / 11.23 \\
\ldots\end{array}$ & $\ldots$ & $\cdots$ \\
\hline V739 Sgr & $2008 / 04 / 25$ & $\begin{array}{l}2008 / 04 / 24 \\
2008 / 05 / 04\end{array}$ & $\begin{array}{l}12.41 / 11.33 / 10.33 \\
12.42 / 11.66 / 10.45\end{array}$ & $2008 / 04 / 29$ & $10.11 / 9.49 / 8.70 / \cdots$ \\
\hline VZ Sgr & $2008 / 04 / 25$ & $\ldots$ & $\ldots$ & & \\
\hline U Aqr & $2008 / 06 / 29$ & $\begin{array}{l}2008 / 06 / 26 \\
2008 / 07 / 04\end{array}$ & $\begin{array}{l}11.40 / 10.74 / 10.23 \\
11.50 / 10.81 / 10.30\end{array}$ & $\cdots$ & $\cdots$ \\
\hline MACHOJ181933 & $2008 / 04 / 25$ & $\begin{array}{l}2008 / 04 / 24 \\
2008 / 05 / 04\end{array}$ & $\begin{array}{l}13.93 / 12.78 / 11.64 \\
13.97 / 12.86 / 11.73\end{array}$ & & \\
\hline $\begin{array}{l}\text { ES Aql } \\
\text { FH Sct }\end{array}$ & $\begin{array}{l}2008 / 05 / 27 \\
2008 / 06 / 02\end{array}$ & $\begin{array}{l}2008 / 05 / 30 \\
2008 / 06 / 03\end{array}$ & $\begin{array}{l}12.28 / 11.17 / 10.09 \\
13.00 / 12.90 / 11.99\end{array}$ & & \\
\hline SU Tau & $2008 / 04 / 28$ & $2008 / 04 / 23$ & $9.79 / 9.15 / 8.56$ & $2008 / 03 / 19$ & $7.72 / 7.28 / 6.74 / 4.96$ \\
\hline DY Per & $2008 / 09 / 13$ & $\ldots$ & $\ldots$ & & \\
\hline V517 Oph & $2008 / 04 / 25$ & $\begin{array}{l}2008 / 04 / 24 \\
2008 / 05 / 04\end{array}$ & $\begin{array}{l}14.05 / 12.51 / 10.96 \\
13.73 / 12.25 / 10.74\end{array}$ & $2008 / 0429$ & $9.74 / 8.45 / 7.08 / 5.22$ \\
\hline
\end{tabular}

Notes.

a Optical and near-infrared photometry for the RCB stars observed with Spitzer by us (Program 50212).

$\mathrm{b}$ The VRI magnitude errors are estimated to be of the order of \pm 0.15 mag (see the text).

the frequency of declines is Jurcsik (1996) who compiled the inter-fade periods for a majority of our sample. She defines a fading of an RCB to be "an initial drop of about 1 mag from a maximum light, independently of the duration and complexity of the minima." The AAVSO Web site provides a historical record of the light curves of many of our RCBs from which we also estimate the frequency of declines. In addition, the ASAS $-3^{8}$ Web site provides nine-year light curves in the $V$ band for many stars in our sample, covering the epoch of the Spitzer observations.

\subsection{Individual Stars}

UV Cas. UV Cas is very rarely seen in decline. Zavatti (1975), from a sparse data set assembled from the literature, found "during 69 years of observations only one deep minimum," a minimum of about four magnitudes recorded more than 80 years ago. An excellent data set from the AAVSO extending back to the 1950s shows no deep declines in last 60 years. There is evidence for a $1.2 \mathrm{mag}$ decline between 1954 August and 1956 August and perhaps one or two even weaker declines but none for the past 30 years. Jurcsik (1996) gives the inter-fade period as 25,500 days; among her sample of $27 \mathrm{RCBs}$ only XX Cam at 36,000 days fades less frequently.

VRI photometry (Table 2) was obtained at the time of the Spitzer observations. We take $U B V$ photometry from Fernie et al. (1972); the star is only slightly variable in $U B V$. Two Micron All Sky Survey (2MASS) JHK magnitudes are adopted (Cutri et al. 2003). A valuable set of JHKLM photometry from 1984 to 2009 is provided by Bogdanov et al. (2010).

\footnotetext{
8 See http://www.astrouw.edu.pl/asas/
}

The Spitzer spectrum shows the interstellar $9.7 \mu \mathrm{m}$ silicate absorption band which is almost entirely removed when the spectrum is corrected assuming $E(B-V)=0.9$. Rao (1980) estimated $E(B-V)=1.0$ from interstellar reddening maps (Fitzgerald 1968) and the assumption that this luminous star must be beyond the majority of the reddening.

Corrected for interstellar reddening, the UBVRI magnitudes are fitted by a $7200 \mathrm{~K}$ blackbody, close to the effective temperature found from optical spectroscopy by Asplund et al. (2000). The 2MASS JHK fluxes imply a brighter blackbody by about 0.2 mag. The Spitzer fluxes are fitted with the principal contribution from a $510 \mathrm{~K}$ blackbody and minor contributions from the Planck tail of the stellar blackbody and a colder $(180 \mathrm{~K})$ blackbody (Figure 6, left-hand panel, and Table 3). The covering factor $R$ sums to 0.035 for the two cool blackbodies (Table 3 ), one of the lowest $R$ for the entire sample. Also, the $6-10 \mu \mathrm{m}$ excess emission is very weak and dependent on the correction for interstellar extinction.

UV Cas shows a large flux variation between IRAS and Spitzer observations. The IRAS $12 \mu \mathrm{m}$ and $25 \mu \mathrm{m}$ fluxes are factors of six (the highest value for our sample) and three (the second highest value for our sample), respectively, greater than the Spitzer values. A fit to the IRAS fluxes and ground-based photometry requires a blackbody at about $800 \mathrm{~K}$ and a higher $R(=0.28)$ than required by the Spitzer fluxes (Figure 6, righthand panel).

Bogdanov et al.'s (2010) survey shows that at the time of the IRAS observations UV Cas was unusually bright in the infrared. The $L$ magnitude was almost 2 mag brighter than when the star was observed by Spitzer and 0.8 mag brighter than the 1973 measurement reported by Rao (1980). The $M$ 
Table 3

Blackbody Fits to the RCBs' SEDs

\begin{tabular}{|c|c|c|c|c|c|c|c|c|c|c|c|}
\hline RCB Star & $\begin{array}{c}T_{\text {star }} \\
(\mathrm{K})\end{array}$ & $\begin{array}{c}T_{B B 1} \\
(\mathrm{~K}) \\
\text { Spitzer }\end{array}$ & $R_{B B 1}$ & $\begin{array}{c}T_{B B 2} \\
(\mathrm{~K})\end{array}$ & $R_{B B 2}$ & $\begin{array}{c}T_{B B 1} \\
(\mathrm{~K}) \\
I R A S\end{array}$ & $R_{B B 1}$ & $\begin{array}{c}T_{B B 2} \\
(\mathrm{~K})\end{array}$ & $R_{B B 2}$ & $E(B-V)^{\mathrm{a}}$ & $\begin{array}{c}\Delta T^{\mathrm{b}} \\
\text { (days) }\end{array}$ \\
\hline UV Cas & 7200 & 510 & 0.03 & 180 & 0.001 & 800 & 0.28 & $\ldots$ & $\ldots$ & 0.90 & 25500 \\
\hline S Aps & 4200 & 750 & 0.37 & $\ldots$ & $\ldots$ & 750 & 0.42 & $\ldots$ & $\ldots$ & 0.05 & 1400 \\
\hline SV Sge & 4200 & 565 & 0.05 & 350 & 0.024 & 720 & 0.15 & $\ldots$ & $\ldots$ & 0.72 & 2500 \\
\hline Z UMi & 5200 & 710 & 0.43 & $\ldots$ & $\ldots$ & 850 & 0.95 & $\ldots$ & $\ldots$ & 0.00 & $\ldots$ \\
\hline V1783 Sgr & 5600 & 560 & 0.28 & $\ldots$ & $\ldots$ & 600 & 0.30 & $\ldots$ & $\ldots$ & 0.42 & $\ldots$ \\
\hline WX CrA & 4200 & 575 & 0.15 & 120 & 0.006 & 700 & 0.49 & $\ldots$ & $\ldots$ & 0.06 & 2000 \\
\hline V3795 Sgr & 8000 & 610 & 0.31 & $\ldots$ & $\ldots$ & 720 & 0.54 & $\ldots$ & $\ldots$ & 0.79 & 6000 \\
\hline V1157 Sgr & 4200 & 770 & 0.59 & 120 & 0.007 & 850 & 1.01 & $\ldots$ & $\ldots$ & 0.30 & $\ldots$ \\
\hline Y Mus & 7200 & 395 & 0.01 & $\ldots$ & $\ldots$ & 590 & 0.07 & $\ldots$ & $\ldots$ & 0.50 & 15300 \\
\hline V739 Sgr & 5400 & 640 & 0.59 & 100 & 0.005 & 900 & 0.64 & 700 & 0.228 & 0.50 & $\ldots$ \\
\hline VZ Sgr & 7000 & 700 & 0.17 & 140 & 0.008 & 700 & 0.17 & 140 & 0.008 & 0.30 & 1300 \\
\hline U Aqr & 5000 & 475 & 0.23 & 140 & 0.021 & 560 & 0.37 & $\ldots$ & $\ldots$ & 0.05 & 1850 \\
\hline MACHOJ181933 & 4200 & 695 & 0.48 & 140 & 0.022 & $\ldots$ & $\ldots$ & $\ldots$ & $\ldots$ & 0.50 & $\ldots$ \\
\hline ES Aql & 4500 & 700 & 0.49 & $\ldots$ & $\ldots$ & 700 & 0.49 & $\ldots$ & $\ldots$ & 0.32 & $\ldots$ \\
\hline FH Sct & 6250 & 540 & 0.10 & 140 & 0.002 & 390 & 0.04 & $\ldots$ & $\ldots$ & 1.00 & $\ldots$ \\
\hline SU Tau & 6500 & 635 & 0.45 & $\ldots$ & $\ldots$ & 635 & 0.50 & $\ldots$ & $\ldots$ & 0.50 & 1200 \\
\hline DY Per & 3000 & 1400 & 0.31 & $\ldots$ & $\ldots$ & 1400 & 0.31 & $\ldots$ & $\ldots$ & 0.48 & $\ldots$ \\
\hline V517 Oph & 4100 & 850 & 0.84 & $\ldots$ & $\ldots$ & 850 & 0.98 & $\ldots$ & $\ldots$ & 0.50 & $\ldots$ \\
\hline $\mathrm{V} \mathrm{CrA}{ }^{\mathrm{c}}$ & 6500 & 550 & 0.38 & 150 & 0.020 & 1600 & 0.23 & 900 & 0.370 & 0.14 & 900 \\
\hline RZ Nor & 5000 & 700 & 0.53 & 320 & 0.035 & 700 & 0.53 & 300 & 0.040 & 0.50 & 1100 \\
\hline RT Nor & 6700 & 320 & 0.01 & 130 & 0.001 & 500 & 0.11 & $\ldots$ & $\ldots$ & 0.39 & 1950 \\
\hline RS Tel & 6750 & 720 & 0.25 & 130 & 0.005 & 620 & 0.22 & $\ldots$ & $\ldots$ & 0.17 & 1200 \\
\hline V482 Cyg & 4800 & 500 & 0.03 & 100 & 0.001 & 650 & 0.09 & $\ldots$ & $\ldots$ & 0.50 & 3400 \\
\hline MV Sgr & 15400 & 1500 & 0.33 & 205 & 0.180 & 1500 & 0.33 & 235 & 0.236 & 0.43 & 6900 \\
\hline RY Sgr & 7200 & 675 & 0.20 & $\ldots$ & $\ldots$ & 870 & 0.76 & $\ldots$ & $\ldots$ & 0.00 & 1400 \\
\hline V854 Cen & 6750 & 900 & 0.32 & 140 & 0.030 & 1100 & 1.00 & $\ldots$ & $\ldots$ & 0.07 & 370 \\
\hline UW Cen ${ }^{\mathrm{d}}$ & 7500 & 630 & 0.44 & 120 & 0.013 & 630 & 0.44 & 150 & 0.033 & 0.32 & 1100 \\
\hline DY Cen & 19500 & 272 & 0.09 & $\ldots$ & $\ldots$ & 330 & 0.10 & $\ldots$ & $\ldots$ & 0.47 & 6400 \\
\hline $\mathrm{R} \mathrm{CrB}$ & 6750 & 950 & 0.30 & $\ldots$ & $\ldots$ & 680 & 0.20 & $\ldots$ & $\ldots$ & 0.00 & 1100 \\
\hline V348 Sgr & 20000 & 707 & 0.63 & 100 & 0.035 & 707 & 0.63 & 100 & 0.035 & 0.45 & 560 \\
\hline HV $2671^{\mathrm{e}}$ & 20000 & 590 & 0.36 & 150 & 0.268 & $\ldots$ & $\ldots$ & $\ldots$ & $\ldots$ & 0.15 & $\ldots$ \\
\hline
\end{tabular}

Notes.

a See the text for more details about the adopted $E(B-V)$ values.

b Inter-fade periods from Jurcsik (1996) (see also the text for more details).

c An additional $550 \mathrm{~K}$ blackbody with a covering factor of 0.37 is needed to fit the IRAS photometry.

d An additional very cool blackbody of $50 \mathrm{~K}$ with a covering factor of 0.05 is needed to fit the Spitzer data.

e An additional very cool blackbody of $40 \mathrm{~K}$ with a negligible covering factor is needed to fit the Spitzer data.

magnitude was about 1.5 mag brighter than in 2008. This IR excess observed by IRAS decayed over about 2000 days and was followed much later by two minor increases by about 0.6 mag in $L$ for a duration of about 1000 days without a pronounced optical decline. Evidently, UV Cas is an irregular infrared variable without contemporary optical variability. Bogdanov et al.'s $L$ and $M$ magnitudes are quite well reproduced by the fit to the IRAS fluxes (Figure 6, right-hand panel).

S Aps. S Aps is a cool RCB with a slightly lower than average tendency to go into decline; Jurcsik (1996) gives the inter-fade period as 1400 days.

The UBVRIJHKLMN magnitudes were assembled from the following sources: $U B V$ (Zhilyaev et al. 1978), UBVRI (Marang et al. 1990), JHKL (Table 2), and $M N$ (Kilkenny \& Whittet 1984). The adopted reddening is $E(B-V)=0.05$ (Asplund et al. 1997). Feast et al. (1997) estimated $E(B-V)=0.13$ but at such low reddenings the correction to the Spitzer and IRAS fluxes is unimportant.

A blackbody fit to the dereddened optical photometry gives a stellar temperature of 4200 K. Dereddened Spitzer fluxes and the contemporaneous $J H K L$ photometry are well fitted with the stellar $4200 \mathrm{~K}$ and a dust blackbody at $750 \mathrm{~K}$ with dust dominating the star at wavelengths at the $K$-band and beyond. The covering factor $R=0.37$ is a typical value.

$\mathrm{S}$ Aps is a striking example where not only are the IRAS and Spitzer fluxes very similar but where measures of the IR excess at other times indicate an almost invariant excess and suggest a circumstellar environment containing a large number of dust clouds. For example, earlier photometry at $K L$ (Glass 1978; Feast et al. 1997) and MN (Kilkenny \& Whittet 1984) are reproduced satisfactorily by the stellar-dust blackbody combination. Variations of no more than several tenths of a magnitude at $L$ are indicative of only minor variations in the cloud population in the circumstellar environment.

SV Sge. SV Sge is a cool RCB experiencing declines at a typical rate; Jurcsik (1996) gives the inter-fade period as 2500 days. Spitzer observed the star at maximum (Table 2).

The UBVRI magnitudes for maximum light are taken as follows: VRI (Table 2), JHK (2MASS), and a $B$ magnitude by assuming a $(B-V)$ identical to that of the $\mathrm{HdC}$ star HD 137613 because the HdC and SV Sge have similar $K$-band spectra (García-Hernández et al. 2010a). A reddening 


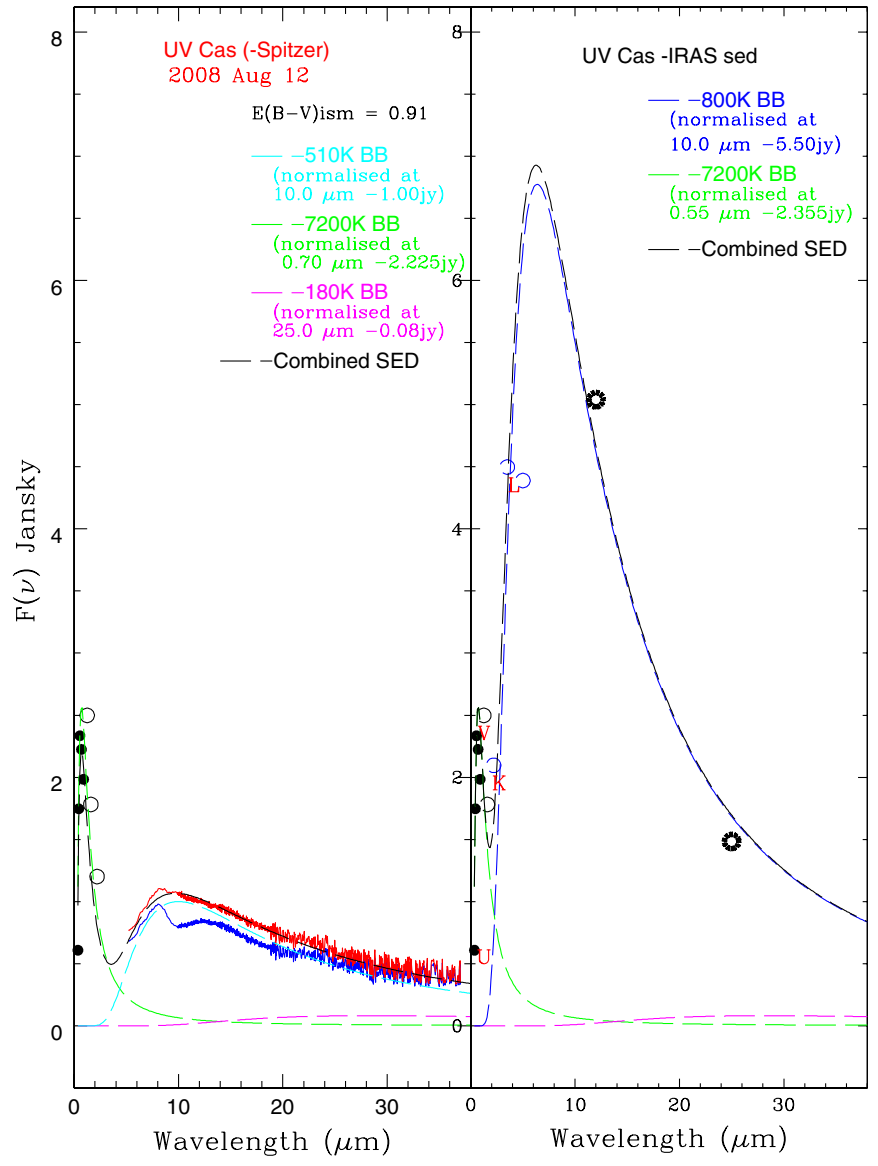

Figure 6. Blackbody fits for UV Cas. The left-hand panel shows a fit to the stellar fluxes computed from reddening-corrected $U B V R I$ ( $U B V$ : Fernie et al. 1972; RI: this work) (black dots), 2MASS JHK (black open circles), and the Spitzer spectrum (corrected for interstellar reddening, in red). The observed Spitzer spectrum (in blue) and the blackbody temperatures are also shown. The right-hand panel shows a fit to the stellar $U B V R I$ and 2MASS $J H$ fluxes and IRAS $12 \mu \mathrm{m}$ and $25 \mu \mathrm{m}$ fluxes with, in addition, the $K L M$ fluxes (blue open circles) estimated from Bogdanov et al. (2010) for the IRAS epoch. Selected $U V K L$ fluxes are labeled for convenience.

$E(B-V)=0.72$ is adopted; a larger reddening results in an emission bump at $9.7 \mu \mathrm{m}$, a feature shown by no other RCB.

The SED is well fitted with a stellar blackbody of $4200 \mathrm{~K}$ and dust blackbodies of $565 \mathrm{~K}$ and $350 \mathrm{~K}$ which in total correspond to a covering fraction $R=0.074$. The IRAS $25 \mu \mathrm{m}$ flux agrees well with the Spitzer flux but the $12 \mu \mathrm{m}$ flux is almost double the Spitzer value which demands a hotter blackbody $(720 \mathrm{~K})$, a larger covering factor $R=0.15$ with the same stellar blackbody. This flux increase at the time of the IRAS measurement implies a rather fresh ejection of dust. Perhaps, this ejection was responsible for the three magnitude optical decline which began in 1981 January and ended in 1982 November. The dust emission contributes a few percent of the Kelvin flux at the time of the Spitzer observation but approaches $10 \%$ at the time of the IRAS observations.

Z UMi. This cool star identified as an RCB by Benson et al. (1994) is frequently in decline; since the star was put on the AAVSO program, it has shown nine declines in 16 years. Kipper \& Klochkova's (2006) analysis led them to suggest Z UMi is a minority RCB of low metallicity with a lithium excess (Goswami et al. 1997). Jurcsik (1996) did not include Z UMi in her determination of inter-fade periods. Spitzer observations were obtained as Z UMi was about two magnitudes below maximum light and recovering from the deepest longest lasting decline on record. The star is at a Galactic latitude of $33^{\circ}$ and, therefore, we assume negligible interstellar reddening.

The stellar blackbody temperature is taken as $5200 \mathrm{~K}$, a value consistent with the spectroscopic estimate of $5250 \pm 150 \mathrm{~K}$ (Kipper \& Klochkova 2006). Photometry for BVI from AAVSO and $J H K$ from 2MASS at maximum light is fitted by this blackbody. A fit to the Spitzer spectrum calls for a blackbody at $710 \mathrm{~K}$ and a covering factor $R=0.43$. Since the optical depth of the cloud(s) along the line of sight likely varies with wavelength, the adopted fit underestimates the (small) stellar contribution at infrared wavelengths.

The IRAS fluxes about $60 \%$ greater than Spitzer values suggest a blackbody at $850 \mathrm{~K}$ and a covering factor $R=0.95$, a value higher than from the Spitzer fluxes at a time when the star was below maximum light.

V1783 Sgr. At the time of the Spitzer observation, V1783 Sgr was near maximum light following the deepest decline in 20 years, a decline that began about 2002 October and ended with restoration to maximum light about 2007 July. Apart from this unusually long but not particularly deep (about three magnitudes) decline, V1783 Sgr has shown only three declines over two decades.

Photometry obtained almost simultaneously with the Spitzer observations is in Table 2. Extensive UBVRI photometry at maximum light was reported by Lloyd Evans et al. (1991) who proposed V1783 Sgr as a cool RCB. The VRI magnitudes in Table 2 are within the range reported by Lloyd Evans et al. An interstellar $E(B-V)=0.42$ is suggested by the elimination of the absorptions at $9.7 \mu \mathrm{m}$ and $18 \mu \mathrm{m}$ from the Spitzer spectrum.

By combining Lloyd Evans et al.'s photometry with the $J H K$ photometry from Table 2 and correcting for the interstellar reddening, the SED is fitted with a stellar blackbody temperature of $5600 \mathrm{~K}$ and dust blackbody of $560 \mathrm{~K}$ for a covering factor of $R=0.28$. The color-corrected IRAS $12 \mu \mathrm{m}$ and $25 \mu \mathrm{m}$ fluxes are similar to the Spitzer values, giving a slightly hotter dust blackbody of $600 \mathrm{~K}$ with a covering factor of $R=0.30$.

The 2MASS $J H K$ is about a magnitude brighter than values in Table 2, and since the star was at visual maximum at the time of the 2MASS observation, it would appear that warm dust was present but off the line of sight.

$W X$ CrA. This cool RCB observed by Spitzer at maximum light experiences declines at a typical frequency; Jurcsik (1996) gives the inter-fade period as 2000 days. Maximum light $U B V R I$ photometry was taken from Marang et al. 1990). The 2MASS $J H K$ photometry agrees well with earlier measurements by Feast et al. (1997). Feast et al.'s $L$ magnitude and Kilkenny \& Whittet's (1984) $M$ and $N$ magnitudes complete the available photometry at maximum light. Interstellar reddening is slight: $E(B-V)=$ 0.06 (Rao 1995 in Asplund et al. 1997; Feast et al. 1997).

A fit to the maximum light reddening-corrected photometry and the Spitzer spectrum calls for a stellar blackbody at $4200 \mathrm{~K}$ and dust at $575 \mathrm{~K}$ and $120 \mathrm{~K}$ with covering factors of $R=0.15$ and 0.006 , respectively.

WX CrA is one of the few stars in the sample with IRAS fluxes, especially at $12 \mu \mathrm{m}$, that are much greater than Spitzer values. The IRAS fluxes require a blackbody at $700 \mathrm{~K}$ with an $R=0.49$. This also accounts for the $L$ and $N$ magnitudes but not the $M$ magnitude from Kilkenny \& Whittet (1984). Glass's (1978) $M$ magnitude is 0.8 mag fainter and falls close to the fit to the IRAS fluxes. Glass comments: "Long-term variations, not closely associated with visible-region behavior, were observed at L." By extension, we infer these variations occur at longer wavelengths too. 
V3795 Sgr. This star observed by Spitzer at maximum light is a warm "minority" RCB which has undergone only two declines in the last 20 years with each lasting about five years; Jurcsik (1996) gives the inter-fade period as 6000 days, one of the longest in her sample.

VRI and $J H K$ photometry (Table 2) was obtained almost simultaneously with the Spitzer observations. A $B$ magnitude is estimated by combining the $V$ from Table 2 with the $(B-V)$ from Kilkenny et al. (1985). JHK magnitudes from Table 2 and 2MASS are in fair agreement. There are slight differences between these values and those provided by Feast et al. (1997) who also gave an $N$ magnitude. Asplund et al. (1997) estimate $E(B-V)=0.79$ but Feast et al. adopted $E(B-V)=0.45$; here, the higher value is assumed.

The stellar blackbody temperature is set at $8000 \mathrm{~K}$, the effective temperature estimated from spectroscopy by Asplund et al. (2000). A dust temperature of $610 \mathrm{~K}$ and a covering factor $R=0.31$ fit the dereddened Spitzer fluxes. The IRAS $12 \mu \mathrm{m}$ and $25 \mu \mathrm{m}$ fluxes, which are only slightly greater than Spitzer values, require a dust blackbody of $720 \mathrm{~K}$ and a covering factor $R=0.54$. This accounts quite well for the flux at $L$ from Feast et al. (1997) obtained when the star was at maximum. Feast et al.'s $K$ magnitude is 0.5 mag brighter than the 2MASS and Table 2 values suggesting that warm dust affected their measurement at $K$.

V1157 Sgr. This cool RCB (Lloyd Evans et al. 1991) has undergone at least three minima in the last 20 years. According to the more recent ASAS-3 database, V1157 Sgr has experienced at least five minima of more than two magnitudes in the last nine years. When Spitzer observed the star it was about two magnitudes below maximum light (Table 2).

Available photometry is limited to that in Table 2 and the 2MASS $J H K$ results. Adopting the latter as a measure of the star at maximum light and with an $E(B-V)$ of 0.3 estimated from a comparison of colors with those of HdC stars, a fit suggests a stellar blackbody temperature $4200 \mathrm{~K}$ and dust blackbodies at $770 \mathrm{~K}$ and $120 \mathrm{~K}$ with covering factors of $R=0.59$ and 0.007 , respectively. The 2 MASS $K$ magnitude received approximately equal contributions from the star and the dust. The IRAS $12 \mu \mathrm{m}$ and $25 \mu \mathrm{m}$ fluxes, which are greater than the Spitzer fluxes, require dust at $850 \mathrm{~K}$.

Y Mus. Y Mus, a warm RCB, has not experienced a decline in more than 20 years. Jurcsik (1996) gives the inter-fade period as 15,300 days, the third longest in her list. Feast et al. (1997) note a brief decline in 1953 reported by Siedel (1957).

Simultaneous ground-based photometry was not obtained but in light of the star's insistence on remaining at maximum light, photometry in the literature may be used to construct the SED. $U B V R I$ photometry is taken from Kilkenny et al. (1985). JHK 2MASS measurements agree with a single observation by Feast et al. (1997) who also provide an $L$ magnitude. Kilkenny \& Whittet (1984) give $M$ and $N$ from 1983, the IRAS epoch. An interstellar $E(B-V)=0.5$ is adopted (Feast et al. 1997; Asplund et al. 1997).

Reddening-corrected fluxes are well fitted by a stellar blackbody at $7200 \mathrm{~K}$ and a dust blackbody at $395 \mathrm{~K}$ with the low covering factor $R=0.01$ (Figure 7).

Strikingly, the infrared excess from IRAS fluxes is considerably stronger: IRAS $12 \mu \mathrm{m}$ and $25 \mu \mathrm{m}$ fluxes are 4.5 and 2.9 times the Spitzer values, respectively. The $1983 M N$ observations (Kilkenny \& Whittet 1984) span the IRAS fit: $M$ is about $50 \%$ stronger and $N$ is about $20 \%$ weaker than the IRAS fit. $L$ (Feast et al. 1997) is matched by this fit to the IRAS fluxes.

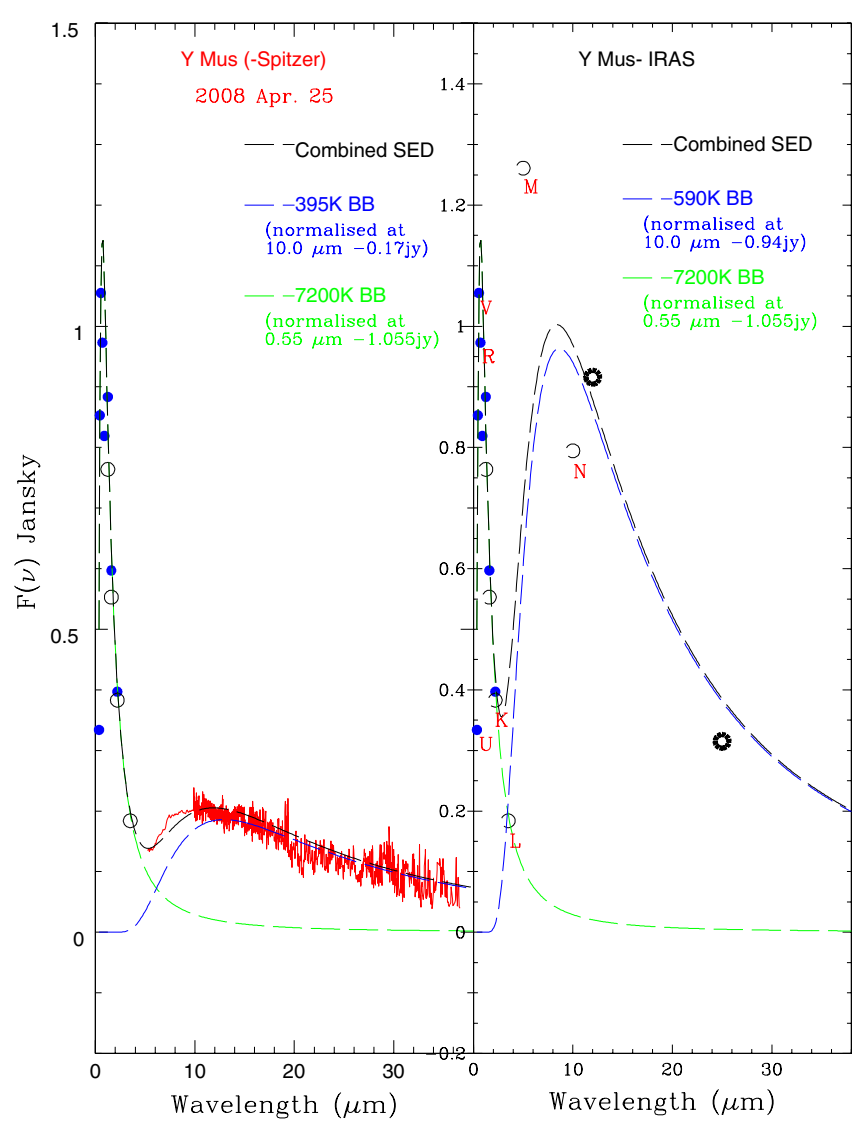

Figure 7. Blackbody fits for Y Mus. The left-hand panel shows a fit to the stellar fluxes computed from reddening-corrected UBVRI (Kilkenny et al. 1985) and $J H K$ 2MASS photometry (blue dots) and JHKL (Feast et al. 1997) (black open circles), and the Spitzer spectrum (corrected for interstellar reddening, in red). The observed Spitzer spectrum (in blue) and the blackbody temperatures are also shown. The right-hand panel shows a fit to the same stellar UBVRIJHK photometric fluxes and IRAS $12 \mu \mathrm{m}$ and $25 \mu \mathrm{m}$ fluxes with, in addition, fluxes at $M$ and $N$ from Kilkenny \& Whittet (1984). Selected $U V R K L M N$ fluxes are labeled for convenience.

Only RT Nor and UV Cas have comparable ratios of IRAS to Spitzer fluxes. The fit to the IRAS fluxes and UBVRIJHKMN photometry gives a dust temperature of $590 \mathrm{~K}$ with a covering factor $R=0.07$.

Evidently, this infrequently declining RCB had an unusually weak circumstellar dust shell at the time of the Spitzer and IRAS observations.

V739 Sgr. This is a cool RCB discovered by Lloyd Evans et al. (1991). The $V$ magnitude (Table 2) agrees very well with the value listed in the ASAS-3 database indicating that the star was at maximum light at the time of the Spitzer observations. Sparse AAVSO measurements of the visual magnitude across 20 years suggest that the star may be a frequent decliner. This is corroborated by the ASAS-3 database, which shows at least four declines in the last nine years.

Photometry is available at VRIJHK from Table 2. The interstellar reddening is assumed to be $E(B-V)=0.5$, the estimate for VZ Sgr in the same direction. A fit to the dereddened photometry and Spitzer fluxes gives a stellar blackbody of $5400 \mathrm{~K}$ with the $K$-band dominated by the dust emission. Spitzer fluxes are well fitted with a $640 \mathrm{~K}$ blackbody with a covering factor $R=0.59$ and there is a hint of a cooler blackbody at $100 \mathrm{~K}$ with $R \simeq 0.005$. The $K$ flux which is not primarily from the star suggests the presence of dust hotter than $640 \mathrm{~K}$. The IRAS $12 \mu \mathrm{m}$ flux is within a few percent of the Spitzer flux. This 


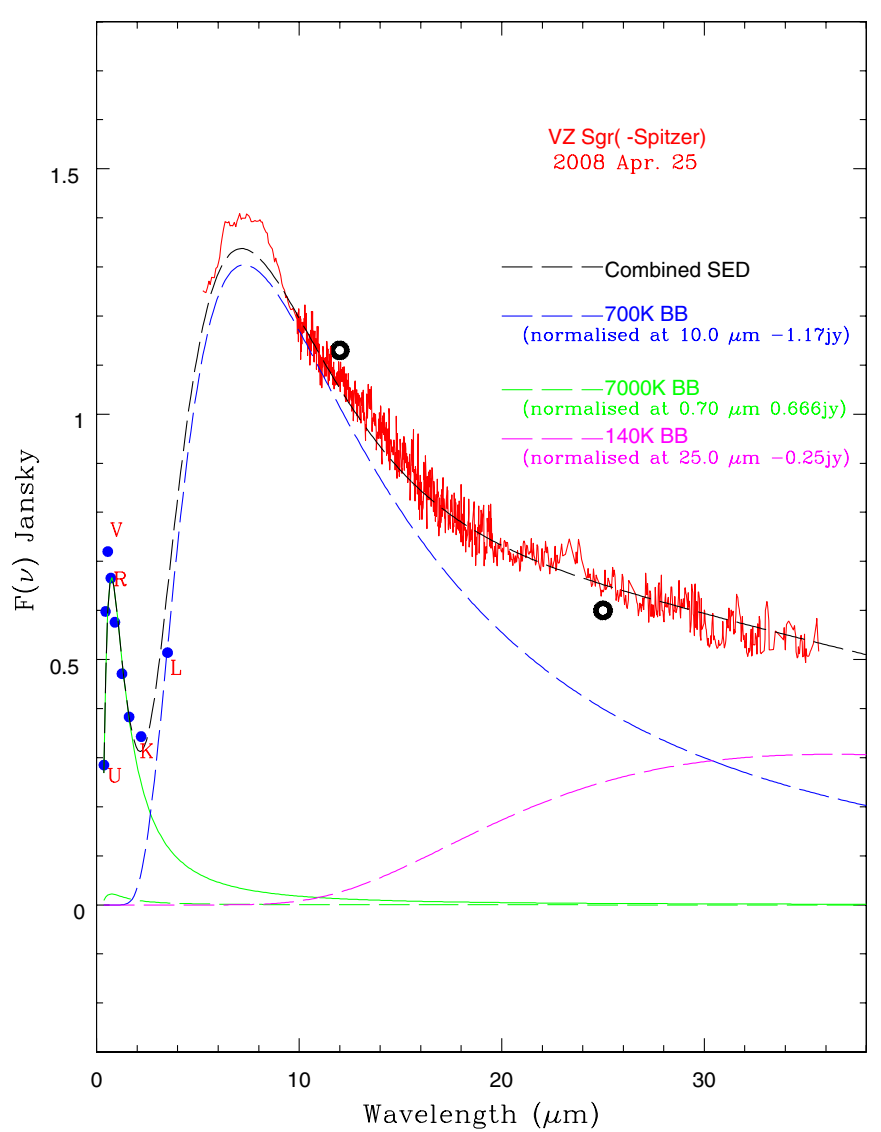

Figure 8. Blackbody fits for VZ Sgr. Reddening-corrected ground-based photometry at UBVRIJHKL (Kilkenny et al. 1985; Feast et al. 1997) and the Spitzer spectrum (in red) are fitted with a stellar (7000 K) blackbody and dust blackbodies at $700 \mathrm{~K}$ and $140 \mathrm{~K}$. Note that the correction for interstellar reddening is negligible for VZ Sgr with $E(B-V)=0.3$ (see the text). IRAS $12 \mu \mathrm{m}$ and $25 \mu \mathrm{m}$ fluxes are also shown and straddle the Spitzer spectrum. Selected $U V R K L$ fluxes are labeled for convenience.

close correspondence and the fact that the $25 \mu \mathrm{m}$ IRAS flux is of low quality makes the fit to the IRAS fluxes more uncertain. Two blackbodies of $900 \mathrm{~K}$ and $700 \mathrm{~K}$ with $R=0.64$ and 0.228 , respectively, can fit the $K$-band flux and the IRAS photometry simultaneously.

VZ Sgr. This warm "minority” RCB has experienced several declines of differing depths in the last 20 years; Jurcsik (1996) gives the inter-fade period as 1300 days. At the time of the Spitzer observations, VZ Sgr was recovering from a deep prolonged decline and still several magnitudes below maximum.

Stellar fluxes for the star at maximum light are taken from the literature: UBVRI (Kilkenny et al. 1985), JHK (Feast et al. 1997; 2MASS), and $L$ (Feast et al. 1997). The interstellar reddening is $E(B-V)=0.30$ (Feast et al. 1997).

The fit to the dereddened photometry and Spitzer fluxes gives a stellar blackbody at the spectroscopic effective temperature of $7000 \mathrm{~K}$ (Asplund et al. 2000) and dust at temperatures of $700 \mathrm{~K}$ and $140 \mathrm{~K}$ with covering factors of $R=0.17$ and 0.008 , respectively (Figure 8). The IRAS $12 \mu \mathrm{m}$ and $25 \mu \mathrm{m}$ fluxes straddle the Spitzer fluxes. Additionally, the $L$ magnitude from 1995 June (Feast et al. 1997) is well matched by the dust's contribution at $700 \mathrm{~K}$ with a small contribution from the star. The $K$ magnitude is slightly contaminated by dust emission. These data suggest that the dusty envelope has maintained a high degree of uniformity over decades.
U Aqr. U Aqr, a cool RCB in the Galactic halo, is distinguished by its extraordinary enrichment of light $s$-process (e.g., $\mathrm{Sr}$ ) nuclides but a near-normal abundance of heavy $s$-process (e.g., Ba) nuclides (Bond et al. 1979; Vanture et al. 1999). Jurcsik (1996) puts the inter-fade period at 1850 days but in the last 20 years U Aqr has spent about 10 years below maximum light. When the Spitzer observations were obtained, U Aqr was about 0.6 mag in $V$ below maximum light in a slow recovery from a deep decline. ${ }^{9}$

The SED is constructed from photometry acquired at maximum light. The interstellar reddening for this halo star about $10 \mathrm{kpc}$ above the Galactic plane (Lawson \& Cottrell 1997) is slight: $E(B-V)=0.05$ (Rao 1995 in Asplund et al. 1997; Feast et al. 1997). Photometry is from the following sources: UBVRI (Lawson et al. 1990; Marang et al. 1990), JHK (2MASS), and JHKL (Feast et al. 1997).

The blackbody combination of $5000 \mathrm{~K}$ for the star and $475 \mathrm{~K}$ and $140 \mathrm{~K}$ for the dust fits the data with covering factors of 0.23 and 0.021 for the cool blackbodies.

The IRAS fluxes straddle the Spitzer spectrum: the IRAS flux at $12 \mu \mathrm{m}$ exceeds its Spitzer counterpart but the IRAS upper limit at $25 \mu \mathrm{m}$ is less than the Spitzer value. A fit to the IRAS data suggests a dust blackbody at $560 \mathrm{~K}$ with a covering factor of 0.37 . This warmer blackbody fits the $L$ magnitude from Feast et al. (1997).

MACHOJ181933. This is a cool RCB discovered by Zaniewski et al. (2005). Comparison of photometry in Table 2 and from the discovery paper shows that the star was at maximum light at the time of the Spitzer observations. Nothing is yet known about the frequency of declines.

VRI (Table 2) and JHK (2MASS) photometry are available. The interstellar reddening is uncertain but not negligible; the star is in the direction of the Galactic Bulge. Zaniewski et al. (2005) put $E(B-V)$ at 1.0. Here, we adopt $E(B-V)=0.5$.

A fit to VRIJHK fluxes and the Spitzer spectrum is obtained with a stellar blackbody of $4200 \mathrm{~K}$ and blackbodies of $695 \mathrm{~K}$ and $140 \mathrm{~K}$ with covering factors of 0.48 and 0.022 , respectively, for the latter two blackbodies. The stellar temperature is similar to that of $\mathrm{S}$ Aps.

ES Aql. ES Aql, a cool RCB (Clayton et al. 2002), declines quite frequently: AAVSO and ASAS-3 observations show a major decline about every year. Not surprisingly, Spitzer caught ES Aql recovering from a deep decline; it was at $V=12.3$ or about 0.8 mag below maximum light. There is no multicolor photometry for ES Aql at maximum light.

$B V R I$ at maximum is inferred from Clayton et al.'s Table 1 and discussion. JHKL magnitudes ${ }^{10}$ are also from Clayton et al. (2002). Adopting an interstellar reddening $E(B-V)=0.32$ (Clayton et al. 2002) and a stellar blackbody of $4500 \mathrm{~K}$ fitted to the dereddened $B V R I$, the Spitzer fluxes are fitted with a $700 \mathrm{~K}$ blackbody and a covering factor of 0.49 (Figure 9). The $L$ magnitude from 1997 June fits the SED composed of the $4500 \mathrm{~K}$ and $700 \mathrm{~K}$ blackbodies; this is expected because for active stars like ES Aql the $L$ magnitude is little affected as a star goes from maximum to minimum. IRAS $12 \mu \mathrm{m}$ and $25 \mu \mathrm{m}$ fluxes are within $10 \%$ of their Spitzer values.

\footnotetext{
9 High-resolution spectra in sample regions of the $K$ band obtained less than two months before the Spitzer observations show stellar molecular absorption features and, therefore, the star not the dust was the dominant contributor to the $K$ band (García-Hernández et al. 2010a).

10 Note that the 2MASS magnitudes of ES Aql are not saturated as stated by Clayton et al. (2002) (G. C. Clayton 2011, private communication).
} 


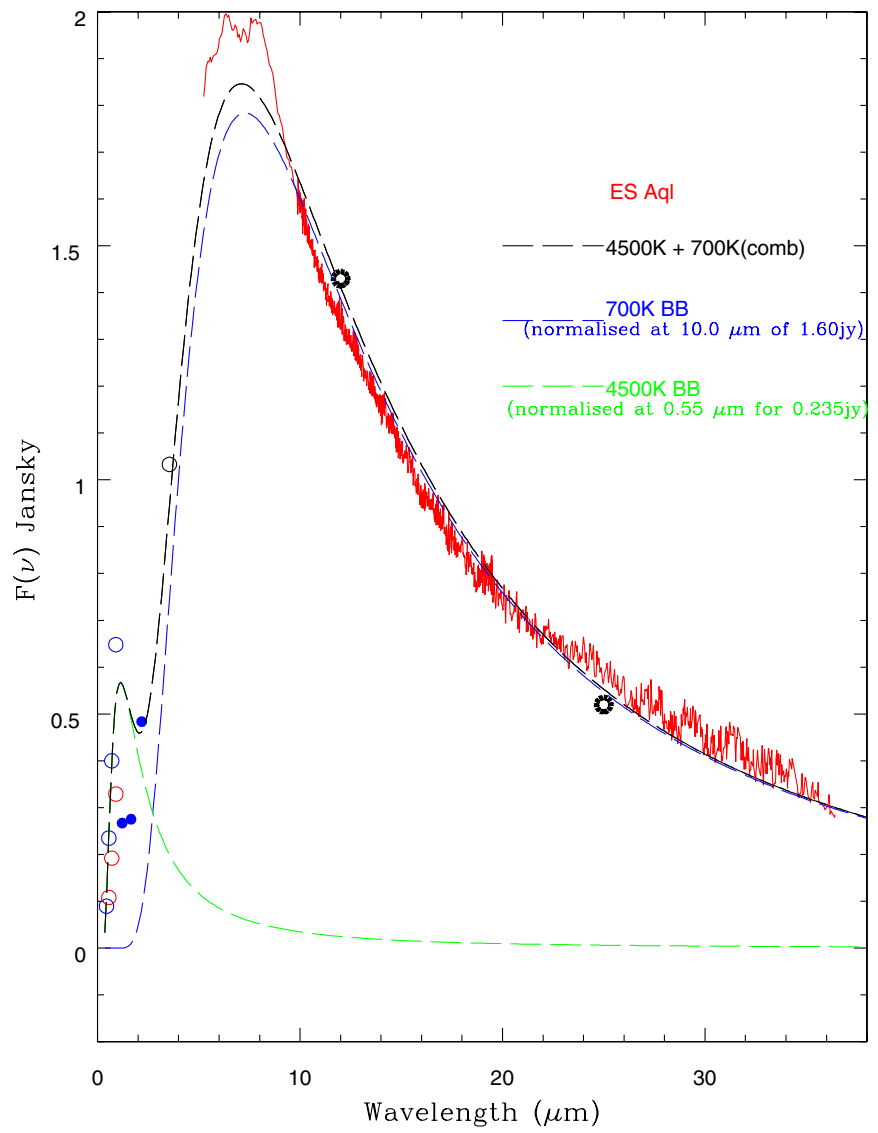

Figure 9. Blackbody fits for ES Aql. Reddening-corrected VRI ground-based photometry (red open circles) and the Spitzer spectrum (corrected for interstellar reddening, in red) are fitted with a stellar $(4500 \mathrm{~K})$ blackbody and a dust blackbody at $700 \mathrm{~K}$. IRAS $12 \mu \mathrm{m}$ and $25 \mu \mathrm{m}$ fluxes are shown and straddle the Spitzer spectrum. Reddening-corrected BVRIJHKL fluxes (see the text) correspond to our VRI ground-based observations (red open circles, Table 2), $B V R I$ at maximum light (blue open circles), 2MASS $J H K$ (blue dots) and $L$ magnitude (black open circle).

FH Sct. This warm RCB has been largely ignored by observers probably because it appears within (but beyond) the Galactic cluster NGC 6694 (M 26). At the time of the Spitzer observations, FH Sct was at 13.00 (Table 2) in agreement with the ASAS-3 database and suggesting that it was about 0.5 mag below maximum.

$V R I$ are taken from Table 2. 2MASS $J H K$ is assumed to refer to maximum light. A reddening $E(B-V)=1.0$ is adopted from a comparison of $(B-V)$ colors of FH Sct with RCBs of similar temperature.

A stellar blackbody of $6250 \mathrm{~K}$ (the spectroscopic effective temperature-Asplund et al. 2000) is adopted and the Spitzer spectrum is fitted by blackbodies of $540 \mathrm{~K}$ and $140 \mathrm{~K}$ with covering factors $R=0.10$ and $R=0.002$, respectively. FH Sct is a rare case where the IRAS $12 \mu \mathrm{m}$ flux is less than the Spitzer flux. The IRAS $25 \mu \mathrm{m}$ flux of moderate quality is similar to the Spitzer value.

$S U$ Tau. This warm RCB frequently experiences declines; SU Tau has been three or more magnitudes below maximum light for nearly half of the last 20 years. Jurcsik (1996) gives the interfade period as 1200 days. Despite its propensity to live below maximum light, the photometry reported in Table 2 shows that it was at maximum light during the Spitzer observations.

The SED was constructed from the following: $U B V$ (Fernie et al. 1972), VRI (Table 2), and JHKL (Table 2). The 2MASS
$J H K$ are 1.4, 1.0, and $0.4 \mathrm{mag}$, respectively, fainter than those in Table 2 but were measured when SU Tau was recovering from a deep decline. Interstellar reddening of $E(B-V)=0.50$ (Glass 1978; Feast et al. 1997) is adopted.

Adopting a stellar blackbody at $6500 \mathrm{~K}$, the spectroscopic effective temperature (Asplund et al. 2000), the Spitzer spectrum, and the $L$ flux are matched with a $635 \mathrm{~K}$ blackbody with a covering factor $R=0.45$. A fit to the $U B V R I J H K$ fluxes gives a stellar blackbody temperature of $6500 \mathrm{~K}$, the spectroscopic effective temperature (Asplund et al. 2000). IRAS $12 \mu \mathrm{m}$ and $25 \mu \mathrm{m}$ fluxes are both slightly higher than Spitzer fluxes.

DY Per. This RCB advertised to be "the coolest metal-poor" RCB (Yakovina et al. 2009) declines at approximately two-year intervals. At the time of the Spitzer observations, DY Per was at $V \simeq 11.8$ or about 0.5 mag below maximum light and at the beginning of a decline that eventually reached a depth of more than five magnitudes below maximum light. Yet it is not very clear if DY Per-like stars really are RCB stars (e.g., Alcock et al. 2001).

We assemble published VBRIJHKLM magnitudes made at or near maximum light (Začs et al. 2007; Alksnis et al. 2009) and adopt the reddening $E(B-V)=0.48$ (Začs et al. 2007). The 2MASS JHK magnitudes are about 0.4 mag fainter than the adopted values, an indication that they were obtained during a decline. ${ }^{11}$

A fit to the dereddened photometry and the Spitzer spectrum with a stellar blackbody at $3000 \mathrm{~K}$ (Yakovina et al. 2009) requires a blackbody at $1400 \mathrm{~K}$ for the dust. The IRAS fluxes are so similar to Spitzer values that a separate fit was not made. With this combination, JHKL are dominated by the dust contribution. The stellar temperature is somewhat hotter than Yakovina et al.'s (2009) estimate of 2900-3100 K from model atmosphere predictions fitted to a 4300-7300 $\AA$ SED but this apparent discrepancy may be due to the presence of strong molecular absorption bands which are not taken into account by a blackbody representation of an SED. More significantly, Tenenbaum et al. (2005) show that the first- and second-overtone CO bands are seen in the $K$ and $H$ bands, respectively, showing that the star dominates the flux in these bands. The $M$-band flux is well below that implied by the $1700 \mathrm{~K}$ blackbody and may be due to strong $\mathrm{CO}$ fundamental band absorption. The IRAS and Spitzer fluxes are very similar.

The Spitzer spectrum has a short wavelength cutoff at $10 \mu \mathrm{m}$ and, therefore, no information is provided about the $6-10 \mu \mathrm{m}$ feature widely seen in RCB spectra. However, DY Per's spectrum shows a sharp emission feature at $11.3 \mu \mathrm{m}$ on a "pedestal" extending from about $10 \mu \mathrm{m}$ to $13.5 \mu \mathrm{m}$.

V517 Oph. Kilkenny et al. (1992) identified V517 Oph as a cool RCB which is "very active in the RCB sense," i.e., frequently in decline. This is corroborated by more recent ASAS-3 measurements, which show several minima in the last nine years. The optical spectrum shown by Kilkenny et al. shows great similarity with S Aps. At the time of the Spitzer observations, the star at $V=14.05$ (Table 2) was about 2.4 mag below maximum light.

Photometry in Table 2 enables the stellar SED to be estimated as it was at the time of the Spitzer observations but it is more valuable for understanding the relation between dust and star to analyze the SED at maximum light. Unfortunately because the star is faint and frequently in decline, measurements at

\footnotetext{
11 Začs et al. report that DY Per has a visual companion, a G0 dwarf at a separation of 2.5 arcsec. This is unlikely to contribute to the SED.
} 
maximum are rare. $U B V$ photometry reported by Kilkenny et al. was, as they noted, obtained below maximum light: their brightest $V=12.4$ may be about one magnitude fainter than maximum light values from the AAVSO and ASAS-3 databases. 2MASS $J H K$ magnitudes are $2.2(J), 1.5(H)$, and $0.8(K)$ mag brighter than those in Table 2. Kilkenny et al. estimate $E(B-V)$ $=0.5-0.6$.

A fit to the 2MASS photometry is possible with a stellar blackbody of $4100 \mathrm{~K}$ and a dust blackbody of $850 \mathrm{~K}$ with a covering factor of 0.84 . This fit accounts quite well for the $L$ flux (Table 2) and a substantial amount of the flux at $K$ is from the dust. The IRAS fluxes are somewhat larger than Spitzer values: $20 \%$ at $12 \mu \mathrm{m}$ and $10 \%$ at $25 \mu \mathrm{m}$ over Spitzer values.

$V$ CrA. This warm minority RCB was observed by Spitzer at maximum light following a deep decline two-and-a-half years previously. The star frequently declines; Jurcsik (1996) gives the inter-fade period as 900 days and only three stars in her sample decline more often.

Photometry at maximum light is taken from the literature: UBVRI (Lawson et al. 1990) and JHKL (Feast et al. 1997; Glass 1978). 2MASS $J H K$ measurements are fainter by $0.2(J), 0.5$ $(H)$, and $0.7(K)$ mag than Feast et al.'s mean values. Kilkenny $\&$ Whittet (1984) measured the $M$ and $N$ magnitudes at a time when the star was at least three magnitudes below its maximum visual brightness. V CrA is only slightly reddened: $E(B-V)$ $=0.14$ (Rao 1995 in Asplund et al. 1997).

A fit to the UBVRI fluxes and the Spitzer fluxes with a $6500 \mathrm{~K}$ stellar blackbody, the spectroscopic effective temperature (Rao \& Lambert 2008), calls for dust blackbodies at $550 \mathrm{~K}$ and $150 \mathrm{~K}$ with covering factors of 0.38 and 0.020 , respectively. This fit does not account for the 2MASS $H K$ and the $L M N$ magnitudes above, all of which are consistently brighter than the Spitzer spectrum. Clearly, the infrared flux varies considerably. The IRAS $25 \mu \mathrm{m}$ flux and the KLMN fluxes, all observed at different times, are reasonably well fitted with a $6500 \mathrm{~K}$ blackbody and dust at $1600 \mathrm{~K}, 900 \mathrm{~K}$, and $550 \mathrm{~K}$ with covering factors of $R=0.23,0.37$, and 0.37 , respectively. However, the IRAS $12 \mu \mathrm{m}$ flux is about $\sim 15 \%$ larger than suggested by our blackbody fitting. This suggests that the dust may be distributed in a disk rather than in a simple discrete cloud or shell. Indeed, Bright et al. (2011) report possible asymmetries in V CrA's circumstellar dust shell from VLTI interferometric data at $10 \mu \mathrm{m}$.

RZ Nor. RZ Nor is a warm RCB notable for the presence of lithium. Jurcsik (1996) gives the inter-fade period as 1100 days, a typical value. According to the ASAS-3 database, the Spitzer observations were obtained when RZ Nor was at maximum light.

Photometry at maximum light is provided by Glass (1978) for $J H K L$, Kilkenny et al. (1985) for UBVRI, and by Feast et al. (1997) for $J H K L$. Kilkenny \& Whittet (1984) observed at $M$ and $N$ when the star was about two magnitudes below maximum. Also, the 2MASS observations were obtained at a minimum when $J$ was 3.8 and $K$ was 0.9 mag fainter than Feast et al.'s estimates for maximum light. A reddening of $E(B-V)=0.5$ is adopted (Glass 1978; Kilkenny \& Whittet 1984; Rao 1995 — see Asplund et al. 1997; Feast et al. 1997).

Fluxes from $U$ to $H$ are fitted by a stellar blackbody of $5000 \mathrm{~K}$, a temperature lower than the spectroscopic effective temperature (Asplund et al. 2000). Spitzer fluxes are fitted with blackbodies of $700 \mathrm{~K}$ and $320 \mathrm{~K}$ with a hint of a $25 \mathrm{~K}$ blackbody introduced to account for a flux increase longward of $34 \mu \mathrm{m}$. The covering factors are 0.53 and 0.035 for the $700 \mathrm{~K}$ and $320 \mathrm{~K}$ blackbodies,

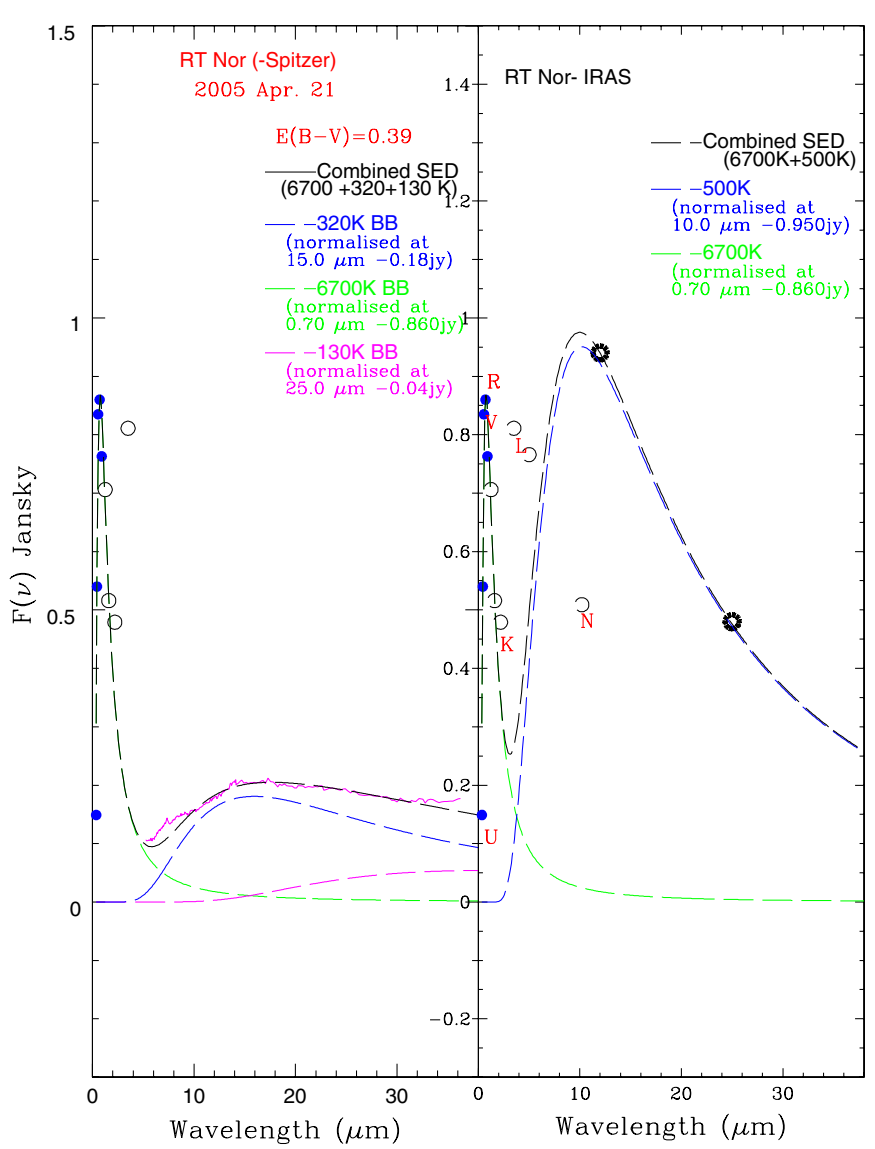

Figure 10. Blackbody fits for RT Nor. The left-hand panel shows a fit to the stellar fluxes computed from reddening-corrected UBVRI (Kilkenny et al. 1985) (blue dots) and JHKL (Feast et al. 1997) (open circles) and the Spitzer spectrum (in red) with the blackbody temperatures shown on the panel. Note that the correction for interstellar reddening is negligible for RT Nor with $E(B-V)$ $=0.39$ (see the text). The right-hand panel shows a fit to the same stellar UBVRIJHKL photometric fluxes and IRAS $12 \mu \mathrm{m}$ and $25 \mu \mathrm{m}$ fluxes with, in addition, fluxes at $M$ and $N$ from Kilkenny \& Whittet (1984). Selected $U V R K L N$ fluxes are labeled for convenience.

respectively. IRAS fluxes for $12 \mu \mathrm{m}$ and $25 \mu \mathrm{m}$ can be fitted with a similar combination of blackbodies at $700 \mathrm{~K}$ and $300 \mathrm{~K}$ and covering factors of 0.53 and 0.040 , respectively.

$R T$ Nor. RT Nor, a warm RCB, was at maximum light when observed by Spitzer. Its last decline occurred about 15 years before. This fact demonstrates the statistical nature of RCB declines because Jurcsik (1996) gives the inter-fade period as short as 1950 days.

Photometry of RT Nor at maximum light is assembled from the literature: UBVRI (Kilkenny et al. 1985), JHKLM (Glass 1978), JHK (2MASS), JHKL (Feast \& Glass 1973), and $M N$ (Kilkenny \& Whittet 1984). Although limited in coverage, the photometry at $L M N$ suggests strong variability. For example, Feast \& Glass (1973) give $L=6.42$ whereas Glass (1978) reports a range from 7.6 to 8.3 from five observations. Similarly, Kilkenny \& Whittet (1984) give $M=5.8$ but Glass measured $M=4.8$. Interstellar reddening of $E(B-V)=0.39$ (Glass 1978; Kilkenny \& Whittet 1984; Rao 1995) is assumed.

A stellar blackbody of $6700 \mathrm{~K}$ and dust blackbodies of $320 \mathrm{~K}$ and $130 \mathrm{~K}$ with remarkably low covering factors of 0.01 and 0.001 , respectively, provide a satisfactory fit to the $U B V R I J K$ and Spitzer fluxes (Figure 10-left-hand panel). It is not surprising that the excess emission at $6-10 \mu \mathrm{m}$ is also unusually weak for RT Nor. 


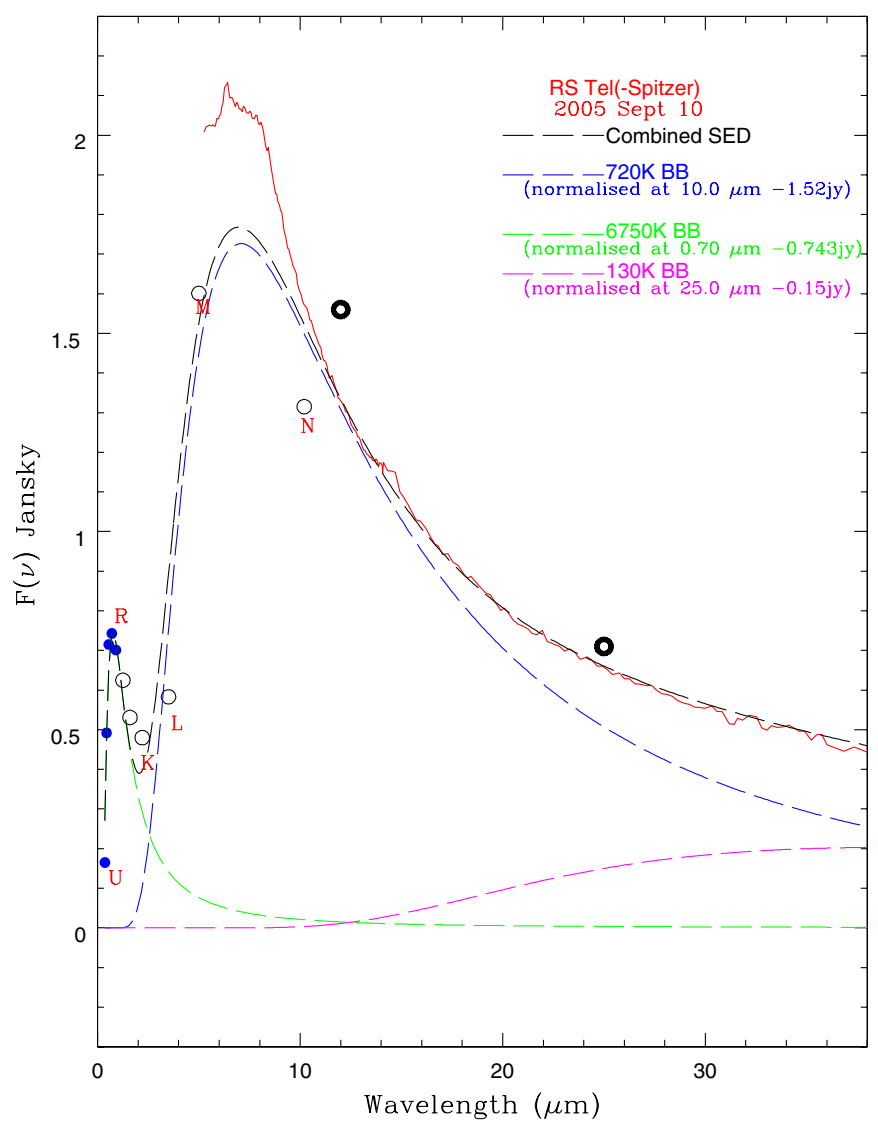

Figure 11. Blackbody fits for RS Tel. Ground-based reddening-corrected photometry at UBVRIJHKL (Kilkenny et al. 1985; Glass 1978; see the text) and the Spitzer spectrum (in red) are fitted with a stellar $(6750 \mathrm{~K})$ blackbody and dust blackbodies at $720 \mathrm{~K}$ and $130 \mathrm{~K}$. Note that the correction for interstellar reddening is negligible for RS Tel with $E(B-V)=0.17$ (see the text). IRAS $12 \mu \mathrm{m}$ and $25 \mu \mathrm{m}$ fluxes and $M N$ from Kilkenny \& Whittet (1984) are also shown. Selected URKLMN fluxes are labeled for convenience.

Dust emission was much stronger at the time of the IRAS observations. The ratio of IRAS to Spitzer fluxes is a factor of five at $12 \mu \mathrm{m}$ and three at $25 \mu \mathrm{m}$. A fit to the $I R A S$ fluxes requires a blackbody of $500 \mathrm{~K}$ and a covering factor of 0.11 (Figure 10, right-hand panel). This fit does not account for the $L M N$ fluxes: the $L M$ fluxes are greater than the $500 \mathrm{~K}$ blackbody but at $N$ the flux is less than that blackbody. A fit solely to the $U B V R I J H K L M N$ fluxes calls for the stellar blackbody to be accompanied by a dust blackbody of $920 \mathrm{~K}$ with a covering factor of 0.11 . The limited observational data suggest that RT Nor experienced an increase in its infrared excess around 1980.

$R S$ Tel. RS Tel, a cool RCB, was observed by Spitzer shortly before it underwent a prolonged decline. This decline apart, RS Tel has experienced no major declines and only one minor decline of about two and a half visual magnitudes in the last two decades. However, Jurcsik (1996) gives the inter-fade period as 1200 days.

Photometry at maximum light is taken from the literature: UBVRI (Kilkenny et al. 1985); JHKLM (Glass 1978; Goldsmith et al. 1990), JHK (2MASS), JHKL (Feast et al. 1997), and $M N$ (Kilkenny \& Whittet 1984). The interstellar reddening is taken to be $E(B-V)=0.17$ (Feast et al. 1997; Kilkenny \& Whittet 1984; Rao 1995 — see Asplund et al. 2000; Bergeat et al. 1999).

Optical and Spitzer fluxes are well fitted by a stellar blackbody of $6750 \mathrm{~K}$, the spectroscopic effective temperature, and blackbodies of $720 \mathrm{~K}$ and $130 \mathrm{~K}$ with covering factors of 0.25 and 0.005 , respectively (Figure 11).
The IRAS $12 \mu \mathrm{m}$ and $25 \mu \mathrm{m}$ fluxes are just $10 \%$ greater than Spitzer values, suggesting a slightly cooler dust blackbody of $620 \mathrm{~K}$ and a covering factor of 0.22 . Also, the $L M N$ fluxes are quite well reproduced by the combination of the $6750 \mathrm{~K}$ and $720 \mathrm{~K}$ blackbodies. Since the $L M N$ and IRAS photometry were obtained decades prior to the Spitzer spectrum, the ability of a single fit to match all the observations implies long-term uniformity of the circumstellar envelope.

V482 Cyg. The RCB V482 Cyg has a K5III companion only 6 arcsec away (Gaustad et al. 1988; Rao \& Lambert 1993). The star was at maximum light when observed by Spitzer. It has experienced just two declines in 20 years, one 14 and the other 19 years ago. Jurcsik (1996) gives the inter-fade period as 3400 days.

Photometry at maximum light is assembled from the literature: BVRI (Rosenbush 1995), JHK (2MASS), and JHKL (Gaustad et al. 1988). An interstellar reddening of $E(B-V)=$ 0.5 is adopted (Rao \& Lambert 1993).

The BVRIJHK fluxes are well fitted with a blackbody of $4800 \mathrm{~K}$ which with blackbodies at $500 \mathrm{~K}$ and $100 \mathrm{~K}$ with covering factors of 0.03 and 0.001 , respectively, combine to fit the Spitzer fluxes. The stellar blackbody is considerably cooler than the $6750 \mathrm{~K}$ effective temperature provided by Asplund et al. (2000) from their abundance analysis.

The IRAS $12 \mu \mathrm{m}$ and $25 \mu \mathrm{m}$ fluxes are $80 \%$ and $10 \%$, respectively, greater than Spitzer fluxes. A fit to the BVRIJHK and IRAS fluxes with the $4800 \mathrm{~K}$ stellar blackbody demands a $650 \mathrm{~K}$ blackbody and a covering factor of 0.09 . Neither the IRAS nor the Spitzer fit accounts for the flux at $L$ measured in 1984. This requires a blackbody of about $1800 \mathrm{~K}$, a temperature hotter than the sublimation temperature of carbon soot, which may result from contamination by the $\mathrm{K} 5$ companion.

MV Sgr. This hot RCB star, according to De Marco et al. (2002), falls outside the reach of "current stellar evolutionary models," i.e., the star is not readily attributable to either the FF or DD scenarios. MV Sgr rarely experiences a decline; the last recorded decline was in the 1950s (Hoffleit 1959). Thus, not suprisingly, Spitzer observed the star at maximum light.

Photometry is taken from the following sources: $U B V R I$ (Kilkenny et al. 1985; Goldsmith et al. 1990), JHKLMN (Kilkenny \& Whittet 1984), and JHK (2MASS). De Marco et al. (2002) estimate $E(B-V)=0.43$.

Dereddened $B V R I$ fluxes are well fitted with a blackbody of temperature $15,400 \mathrm{~K}$, the effective temperature estimated from spectroscopy by Jeffery et al. (1988), but other temperatures will fit these fluxes in the Rayleigh-Jeans tail. The Spitzer spectrum is matched with a blackbody at $205 \mathrm{~K}$ and a covering factor of 0.18 . The IRAS fluxes are reproduced by a slightly hotter $235 \mathrm{~K}$ blackbody with a slightly larger covering factor. This increase also fits the $N$ flux but the $M$ flux exceeds the fit by a factor of about $50 \%$.

The limited photometry at JHKLMN indicates the presence of a variable source with a temperature of $1500 \mathrm{~K}$ or so. For example, the $J H K L$ fluxes require in addition to the $15,400 \mathrm{~K}$ and $205 \mathrm{~K}$ blackbodies a blackbody at $1500 \mathrm{~K}$ with a covering factor of 0.33 . A fit to IRAS and $M N$ fluxes requires $1500 \mathrm{~K}$ and $235 \mathrm{~K}$ blackbodies together with the stellar 15,400 K blackbody to fit $B V R I$. This combination predicts too strong a flux at $K$. This contribution at about $1500 \mathrm{~K}$ may come from fresh production of carbon soot.

$R Y$ Sgr. For RY Sgr, a warm RCB, deep declines occur about every four years and the Spitzer observations were made when 
RY Sgr was about three magnitudes below maximum light. Jurcsik (1996) gives the inter-fade period as 1400 days.

The star is represented by a $7200 \mathrm{~K}$ blackbody; the spectroscopic effective temperature is $7250 \mathrm{~K}$ (Asplund et al. 2000). The Spitzer fluxes are fitted with a blackbody of $675 \mathrm{~K}$ and a covering factor of 0.20 .

Fluxes measured by ISO from $4 \mu \mathrm{m}$ to $26 \mu \mathrm{m}$ exceed Spitzer values and suggest a dust blackbody of $820 \mathrm{~K}$ with a covering factor of 0.38. IRAS fluxes which exceed both ISO and Spitzer values are fitted with a dust blackbody of $870 \mathrm{~K}$ and a covering factor of 0.76 . It is tempting in the sequence of declining blackbody temperatures and covering factors from IRAS to ISO to Spitzer to see an evolutionary sequence from IRAS in 1983 to ISO in 1997 and then to Spitzer in 2004 October. In light of the frequency of declines, an evolutionary interpretation is probably too simplistic. Bright et al. (2011) report the apparent presence of asymmetric circumstellar material around RY Sgr.

V854 Cen. V854 Cen, a most active warm RCB, has the shortest known inter-fade period (370 days) of the RCBs studied by Jurcsik (1996). Spitzer observations were obtained when V854 Cen was at a visual magnitude between five and six magnitudes below maximum. An important distinguishing mark is its modest $\mathrm{H}$ deficiency among RCBs: hydrogen is deficient by a factor of only 100-1000 (Asplund et al. 1998).

Published photometry for maximum light is available from Lawson et al. (1999) for UBVRI and Feast et al. (1997) for JHKL. Interstellar reddening $E(B-V)=0.07$ is adopted (Feast et al. 1997).

$B V R I$ is quite well fitted with a blackbody of $6750 \mathrm{~K}$, the spectroscopic effective temperature (Asplund et al. 1998). The Spitzer spectrum is fitted by a blackbody combination of $900 \mathrm{~K}$ and $140 \mathrm{~K}$ with covering factors of 0.32 and 0.03 , respectively. This fit accounts fairly well for the $H K L$ at minimum light, the phase at which V854 Cen was when observed by Spitzer.

IRAS fluxes are stronger than Spitzer values and similar to the $I S O$ values (Lambert et al. 2001). The fit to IRAS (and ISO) fluxes requires a $1100 \mathrm{~K}$ blackbody with a covering factor of unity. The $H K L$ fluxes at optical maximum are dominated by dust emission; the flux increases with increasing wavelength. This fit accounts quite well for the $J H K L$ fluxes at maximum light. Indeed, the ISO observation was made when V854 Cen was only slightly below maximum light. Very recent high angular resolution interferometric observations of V854 Cen show the possible presence of asymmetries in the circumstellar envelope (Bright et al. 2011).

UW Cen. UW Cen, a warm RCB, has an inter-fade period of 1100 days (Jurcsik 1996). A reflection nebula illuminated by the star was discovered by Pollacco et al. (1991) and further studied by Clayton et al. (1999). Spitzer observations were obtained at maximum light during an approximately 18 month restoration to maximum light between an earlier deep decline lasting almost a decade and the next decline which ended early in 2010.

Photometry for maximum light is taken from the literature: UBVRI (Kilkenny et al. 1985; Goldsmith et al. 1990), JHKLM (Glass 1978), JHKL (Feast et al. 1997; Goldsmith et al. 1990), $M N$ (Kilkenny \& Whittet 1984; Goldsmith et al. 1990). Interstellar reddening $E(B-V)=0.32$ is assumed (Glass 1978; Kilkenny \& Whittet 1984; Rao 1995 —see Asplund et al. 1997; Feast et al. 1997).

A fit to the dereddened fluxes with a $7500 \mathrm{~K}$ stellar blackbody, the spectroscopic effective temperature (Asplund et al. 2000), calls for blackbodies at $630 \mathrm{~K}, 120 \mathrm{~K}$ and $50 \mathrm{~K}$ with covering factors of $0.44,0.013$, and 0.05 , respectively. Dust emission extends to the $K$ band; the $K$ flux is greater than that at $H{ }^{12}$ The fit to the Spitzer fluxes accounts well for the $L$ and $M$ fluxes observed about 30 years earlier. The $N$ flux is less than the Spitzer value by about $20 \%$. The IRAS $12 \mu \mathrm{m}$ flux is in good agreement with the Spitzer value but the $25 \mu \mathrm{m}$ flux is about $30 \%$ higher than the Spitzer measurement.

DY Cen. DY Cen, a hot RCB, has not experienced a decline in 20 years, although the maximum $V$ magnitude seems to have fallen by $0.7 \mathrm{mag}$ in 45 years (De Marco et al. 2002). Jurcsik (1996) gives the inter-fade period as 6400 days. Lambert \& Rao (1994), on the basis of the abundance analysis by Jeffery \& Heber (1993), considered DY Cen to be a minority RCB. De Marco et al. (2002) note that DY Cen is likely to be related to the other (cooler) RCBs. The star is unusually H-rich for an RCB: $\mathrm{H} / \mathrm{He}=0.1$ by number (Jeffery \& Heber 1993).

Photometry in the literature provides the following: $U B V$ (Pollacco \& Hill 1991), UBVRI (Kilkenny et al. 1985), JHK (2MASS), and $N$ (Kilkenny \& Whittet (1984). Interstellar reddening $E(B-V)=0.47$ is adopted (Jeffery \& Heber 1993; Rao et al. 1993).

A stellar blackbody of $19,500 \mathrm{~K}$, the effective temperature obtained by Jeffery \& Heber from their spectroscopic analysis, is fitted to the reddening-corrected UBVRIJHK fluxes. Since these bandpasses are in the Rayleigh-Jeans tail of a hot blackbody, other fits are possible. The Spitzer fluxes show that the dust is predominantly cool with a blackbody of $272 \mathrm{~K}$ and a covering factor of 0.09. Emission features from a mixture of PAHs and $\mathrm{C}_{60}$ molecules are prominent (García-Hernández et al. 2011). IRAS fluxes are within about $10 \%$ of the Spitzer values.

$R C r B$. R CrB is the most studied warm RCB. It was at maximum when the Spitzer spectrum was obtained. Jurcsik (1996) gives the inter-fade period as 1100 days.

The UBVRIJ fluxes are fitted with a $6750 \mathrm{~K}$ blackbody, the spectroscopic effective temperature (Asplund et al. 2000). The 10-20 $\mu \mathrm{m}$ Spitzer spectrum is fitted with a $950 \mathrm{~K}$ blackbody with a covering factor of 0.30 . This fit also accounts well for the $K$ and $L$ fluxes where the shell makes the dominant contribution to the latter and the star to the former. The IRAS $12 \mu \mathrm{m}$ and $25 \mu \mathrm{m}$ fluxes are higher than Spitzer values. The $I S O$ spectrum from 1998 January, in fair agreement with the IRAS fluxes, was fitted with a combination of two blackbodies: $1390 \mathrm{~K}$ and 610 K (Lambert et al. 2001). Rao \& Nandy (1986) fitted the IRAS observations with a blackbody at $680 \mathrm{~K}$ and Clayton et al. (1995) fitted an IRAS LRS spectrum with a blackbody at $650 \mathrm{~K}$. $\mathrm{R} \mathrm{CrB}$ is a variable (out of decline) in the infrared. There are variations of about one magnitude at $K$ and of two magnitudes at $L$. Strecker (1975) and Feast et al. (1997) suggest that the $L$ variations follow a period of about 1260 days. Thus, a fit of the infrared-optical SED requires a variable infrared excess.

V348 Sgr. V348 Sgr is "the second most active RCB star (after V854 Cen)" (De Marco et al. 2002); Jurcsik (1996) gives the inter-fade period as 560 days. V348 Sgr is a hot RCB with an effective temperature about 22,000 K (Jeffery 1995). De Marco et al. (2002) suggest with some reservations that V348 Sgr is an FF post-AGB star becoming once again the central star of a planetary nebula. Visible nebulosity is present (Pollacco et al. 1990) supporting the evolutionary interpretation. Spitzer observations were obtained when V348 Sgr was undergoing a minimum and are discussed in more detail in Clayton et al. (2011).

\footnotetext{
12 The fact that dust dominates the spectrum in the $K$ band is confirmed by $K$-band spectra obtained in 2007 February when UW Cen was at maximum light (García-Hernández et al. 2009a).
} 
Photometry at maximum light is assembled as follows: BVRI (Heck et al. 1985), JHKLM (Glass 1978), and JHK (2MASS). Observed fluxes are corrected for an interstellar reddening of $E(B-V)=0.45$ (Pollacco et al. 1990).

Adopting a stellar blackbody at 20,000 K, the Spitzer spectrum is fitted with a combination of $707 \mathrm{~K}$ and $100 \mathrm{~K}$ blackbodies with covering factors of 0.63 and 0.035 , respectively. This fit accounts well for the JHKLM fluxes from the 1970s and the 2MASS JHK from 1998. Also, the IRAS fluxes only slightly exceed their Spitzer counterparts. Rao \& Nandy's (1986) fit to the IRAS 12, 25, 60, and $100 \mu \mathrm{m}$ fluxes required warm dust $(600 \mathrm{~K})$ and cold dust $(60-100 \mathrm{~K})$, a mixture very similar to the present fit to the Spitzer observations obtained nearly 30 years later. This constancy of the infrared emission over decades is not surprising given that V348 Sgr is second most active RCB.

$H V$ 2671. De Marco et al. (2002) note that HV 2671 in the LMC and V348 Sgr have almost identical optical spectra. The Spitzer spectra are, however, very different (Figure 5; see also Clayton et al. 2011 for more details). De Marco et al. provide $J H K$ magnitudes and a reddening estimate $E(B-V)=0.15$. Soszyński et al. (2009) report $V$ and $I$ for maximum light. On the assumption that the stellar blackbody temperature is $20,000 \mathrm{~K}$, the same as for V348 Sgr, the fit to the JHK fluxes and the Spitzer spectrum calls for blackbodies at $590 \mathrm{~K}$ and $150 \mathrm{~K}$ with covering factors of 0.36 and 0.268 , respectively. There is a hint of the presence of an additional and very cool $40 \mathrm{~K}$ dust component with a negligible covering factor. The dust distribution around HV 2671 differs substantially from that around V348 Sgr; this result is not surprising.

\section{PROBING THE CLOUDY CIRCUMSTELLAR ENVIRONMENT}

Early investigations of the infrared emission from RCBs searched for variability of that emission. Among the earliest studies of infrared variability, Forrest et al.'s (1972) observations of $\mathrm{R} \mathrm{CrB}$ through a six-magnitude visual decline showed that the infrared flux from $3.5 \mu \mathrm{m}$ to $11 \mu \mathrm{m}$ remained essentially unaffected by the decline. This result suggested that the dust cloud causing the decline represents a small addition to the warm circumstellar material already in place.

Long-term monitoring of the infrared excess of RCBs potentially offers particular insights into the structure and growth of clouds in the circumstellar environment. In this regard, Feast and colleagues at the SAAO provided a valuable set of observations. For example, Feast et al. (1997) report JHKL photometry of $12 \mathrm{RCBs}$ for timespans of up to 23 years. At $J H K$, the star generally dominated the observed flux but at $L$ the infrared excess from dust is significant. Observations at $L$ along with other evidence led to the proposal that dust is ejected into the circumstellar environment in the form of "random puffs" (Feast 1979, 1986, 1996, 1997; Feast et al. 1997).

It is in the context of the "puff" model that we explore below what may be deduced from the relation between the Spitzer and $I R A S$ fluxes and the dependence of the covering factor $R$ on the frequency of optical declines. These relations suggest that dust is ejected in puffs, often off the line of sight, and very likely in most cases the ejection occurs approximately isotropically off the star (i.e., at random directions).

\subsection{Spitzer versus IRAS}

A comparison of the Spitzer spectra with the IRAS $12 \mu \mathrm{m}$ and $25 \mu \mathrm{m}$ fluxes provides insight into the variability of the emission from circumstellar dust on a timescale of about 25 years. This is potentially valuable because 29 of our RCBs are in the IRAS catalog. In Figure 12, we present histograms of the ratios of the IRAS to Spitzer fluxes at $12 \mu \mathrm{m}$ (left panel) and $25 \mu \mathrm{m}$ (right panel).

With the exception of five outliers, the ratios at $12 \mu \mathrm{m}$ are less than two: the mean value is $r_{12}=1.41 \pm 0.35$ from 22 stars. A correction has to be applied because the IRAS fluxes refer to a broad band and assigned an effective wavelength of $25 \mu \mathrm{m}$. The effective wavelength assumes that the energy distribution has the form $f_{\nu} \propto v^{-1}$ or $f_{\lambda} \propto \lambda^{-1}$. Given that the Spitzer spectra are close approximations to a blackbody spectrum, one may estimate a color correction according to a recipe provided in the IRAS catalog (Beichman et al. 1988). This color correction reduces the IRAS catalog entries by about $16 \%$ or the ratio $r_{12}$ is 1.18 .

Setting aside the same five outliers, the ratio $r_{25}=1.27 \pm 0.32$ from 21 stars: R CrB was not observed by Spitzer at $25 \mu \mathrm{m}$ and the ratios for VZ Sgr and U Aqr are set at the value given by the upper limit to their IRAS $25 \mu \mathrm{m}$ fluxes. After the color correction, this value of $r_{25}$ is not sensibly different from unity.

For stars with extended infrared emission, the IRAS fluxes will be systematically larger than Spitzer values because of the larger IRAS aperture. The IRAS aperture was approximately 2 minutes of arc which is larger than the Spitzer aperture for the SH observations $\left(4^{\prime \prime} .7 \times 11^{\prime \prime} .3\right)$ used at $12 \mu \mathrm{m}$ and for the LH observations $\left(11^{\prime \prime} .1 \times 22^{\prime \prime} .3\right)$ used at $25 \mu \mathrm{m}$. Perhaps coincidentally the $25 \mu \mathrm{m}$ Spitzer fluxes through the larger LH aperture are closer to their IRAS counterparts than the $12 \mu \mathrm{m}$ fluxes through the $\mathrm{SH}$ aperture. As we have already mentioned in Section 2.2, all RCBs in our sample are point-like sources for Spitzer. IRAS and Spitzer fluxes agree very well when astronomical sources are point-like for Spitzer (e.g., GarcíaHernández et al. 2007, 2009b). Indeed, R CrB itself, which is known to be extended, shows no important flux differences between IRAS and Spitzer (also V348 Sgr with a PN of 30"; Clayton et al. 2011). Thus, the presence of the five outliers is not related to a possible extended emission in these sources. ${ }^{13}$ Finally, note that possible extended diffuse background emission is usually due to much colder dust (see, e.g., Cox et al. 2011), which emits at wavelengths longer than $25 \mu \mathrm{m}$ (see, e.g., the case of the hot RCBs V348 Sgr and HV 2671; Clayton et al. 2011).

The IRAS-Spitzer comparison has thus shown that the circumstellar dust emission for the great majority of the RCBs is unchanged over the last couple of decades. This is not a surprising result given the extensive $L$-band photometric measurements on a fair sample of RCBs conducted at the SAAO over several years (Feast et al. 1997). Attention is necessarily drawn to the five outliers. Before discussing this quintet, we note the surprising result that these outliers, all with high values of $r_{12}$ and $r_{25},{ }^{14}$ cannot be matched with a similar number of outliers with remarkably low values of $r_{12}$ and $r_{25}$. Three of the five have $r_{12}$ of between five and six. A comparable outlier on the low- $r$ side of the histogram would have a value of less than 0.2 , but the sole RCB with an $r_{12}$ of less than unity is FH Sct with $r_{12}$ $=0.7$. With respect $r_{25}$, the asymmetry is not quite so severe. The lowest $r_{25}$ values are upper limits of 0.9 and 0.8 for VZ Sgr and $\mathrm{U}$ Aqr, respectively, to be compared with the highest two

\footnotetext{
13 Note also that extended emission is not seen in the available Spitzer/IRAC images at $8 \mu \mathrm{m}$.

14 The $r_{25}$ for WX CrA is 1.8 , a value which does not qualify it as an outlier at $25 \mu \mathrm{m}$.
} 

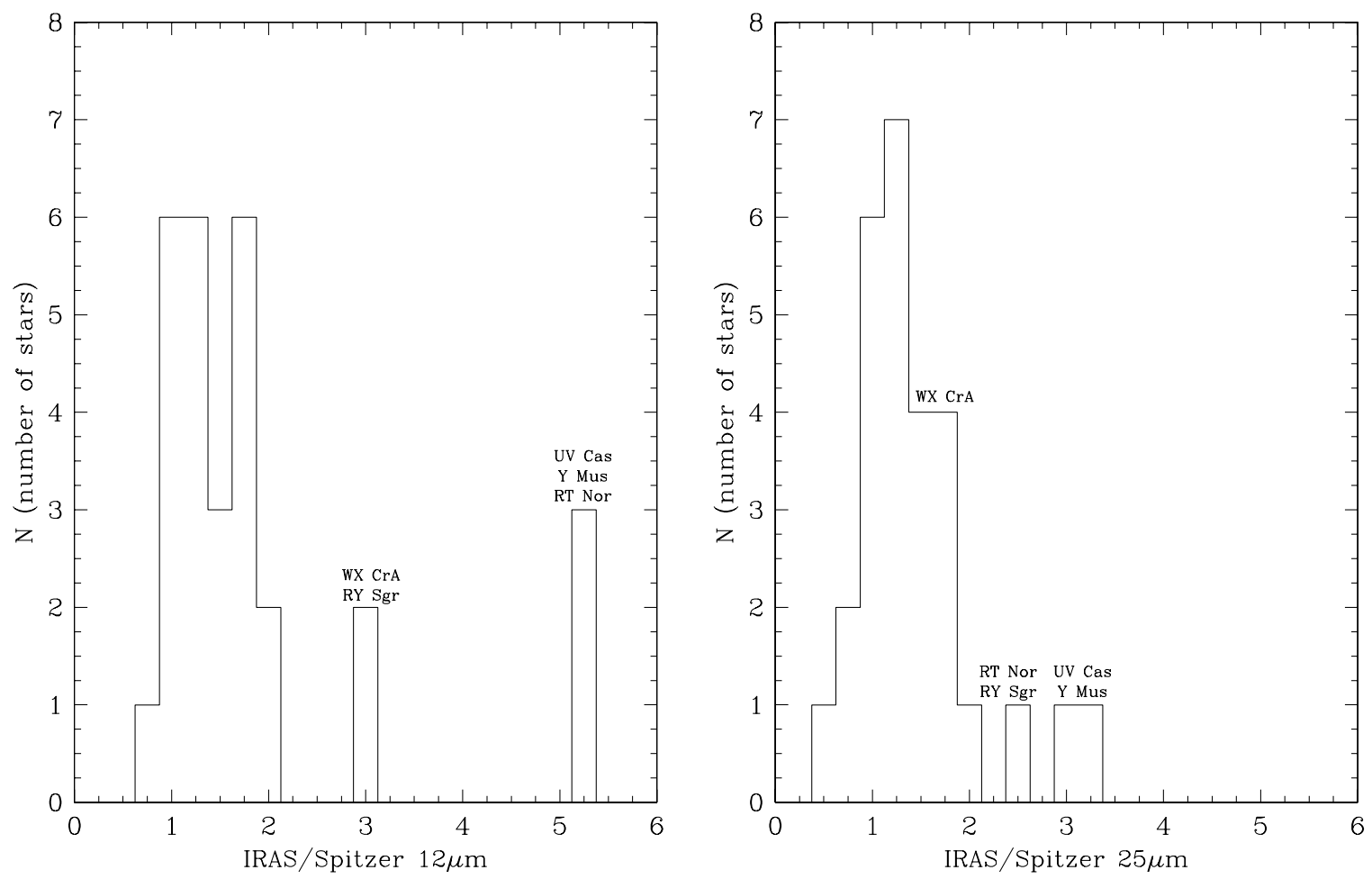

Figure 12. Histograms of the IRAS/Spitzer flux ratios. Left panel—fluxes at $12 \mu \mathrm{m}$. Right panel—fluxes at $25 \mu \mathrm{m}$. Fluxes are uncorrected for interstellar reddening. Color corrections have not been applied to the IRAS fluxes. Five outliers are identified.

values of 3.3 and 2.9 for Y Mus and UV Cas, respectively. Is this presence of outliers on the high- $r$ and the paucity of outliers on the low- $r$ side just a statistical fluke or a hint of a long-term evolution in RCB dust shells? The latter seems unlikely given that the evolutionary timescale is likely to be a few thousand years and the interval between IRAS and Spitzer observations is less than 30 years.

With respect to the outliers, a possible explanation is that these are RCBs which rarely eject dust. Dust ejection rates may or may not be closely related to the frequency of declines, i.e., the formation and presence of dust along the line of sight. Dust formation may or may not occur in preferential directions such as an equatorial plane or as polar plumes. Next, we discuss the five outliers in order of decreasing $r_{12}$ : three-Y Mus, UV Cas, and RT Nor-have $r_{12} \simeq 5$ and two-WX CrA and RY Sgr-have $r_{12} \simeq 3$.

$Y$ Mus. Y Mus has not experienced a decline in the nearly 30 years covered by AAVSO records which begin about 1982 January. The covering factor $R=0.009$ from the Spitzer observations is the lowest among our RCB sample. Even the IRAS fluxes correspond to a low $R=0.07$. Thus, we conclude that Y Mus is simply a poor producer of dust, i.e., the preferred axis for dust production is not orthogonal to the line of sight. One supposes that Y Mus is slightly more active with respect to dust production than XX Cam which has not been observed in decline at all and has no infrared excess out to $10 \mu \mathrm{m}$ (Rao et al. 1980).

UV Cas. At the time of the IRAS observations, UV Cas was considered to be a typically dusty RCB with a covering factor $R=0.28$ but by the time that Spitzer observed it the infrared fluxes had declined sharply and the covering factor had dropped to $R=0.03$. The dust cloud responsible for infrared emission at the time of IRAS did not cause a deep optical decline, of which none have been seen for 60 years. Bogdanov et al.'s
(2010) survey of JHKLM for 25 years from 1984 shows a major IR excess present and weakening at the time of the IRAS observations with two weaker episodes in subsequent years. Bogdanov et al.'s KLM magnitudes from 1984 are acceptably fitted by the blackbody fit to the reddening-corrected IRAS fluxes (Figure 6).

RT Nor. RT Nor closely resembles Y Mus in terms of its low covering factors at the time of the IRAS and Spitzer observations. RT Nor has experienced (according to AAVSO records) only three or four optical declines of three or more magnitudes in the last 50 years. None of the declines happened a few years prior to the IRAS or Spitzer observations. As in the case of Y Mus, we suppose that RT Nor is simply an infrequent producer of dust.

WX CrA. In contrast to Y Mus, UV Cas, and RT Nor, WX $\mathrm{CrA}$ is frequently in decline; for example, in the 13 years from late 1992 to late 2005 it was almost always below maximum light, often by two or more magnitudes. The Spitzer observation acquired at maximum light was preceded by about three years at maximum light. The IRAS observations were similarly acquired at maximum light and preceded by about a decade without optical declines according to AAVSO records and Feast et al. (1997). Thus, the greater IRAS fluxes and higher covering factor ( $R=0.49$ versus $R=0.14$ ) relative to Spitzer fluxes must be due to ejections of dust off the line of sight. Then, the fact that both $R$ values are fairly representative values suggests that ejection of puffs occurs about as frequently as optical declines, i.e., there is no strong directional dependence for ejection of puffs.

$R Y$ Sgr. Infrared emission from RY Sgr provides higher covering factors than found for WX CrA suggesting a higher dust ejection rate. At the time of the Spitzer observations, RY Sgr was in decline but the covering factor $R$ was only 0.20 . An above average $R(=0.76)$ was found from the IRAS observations. Major optical declines occur at a frequency of about one every 


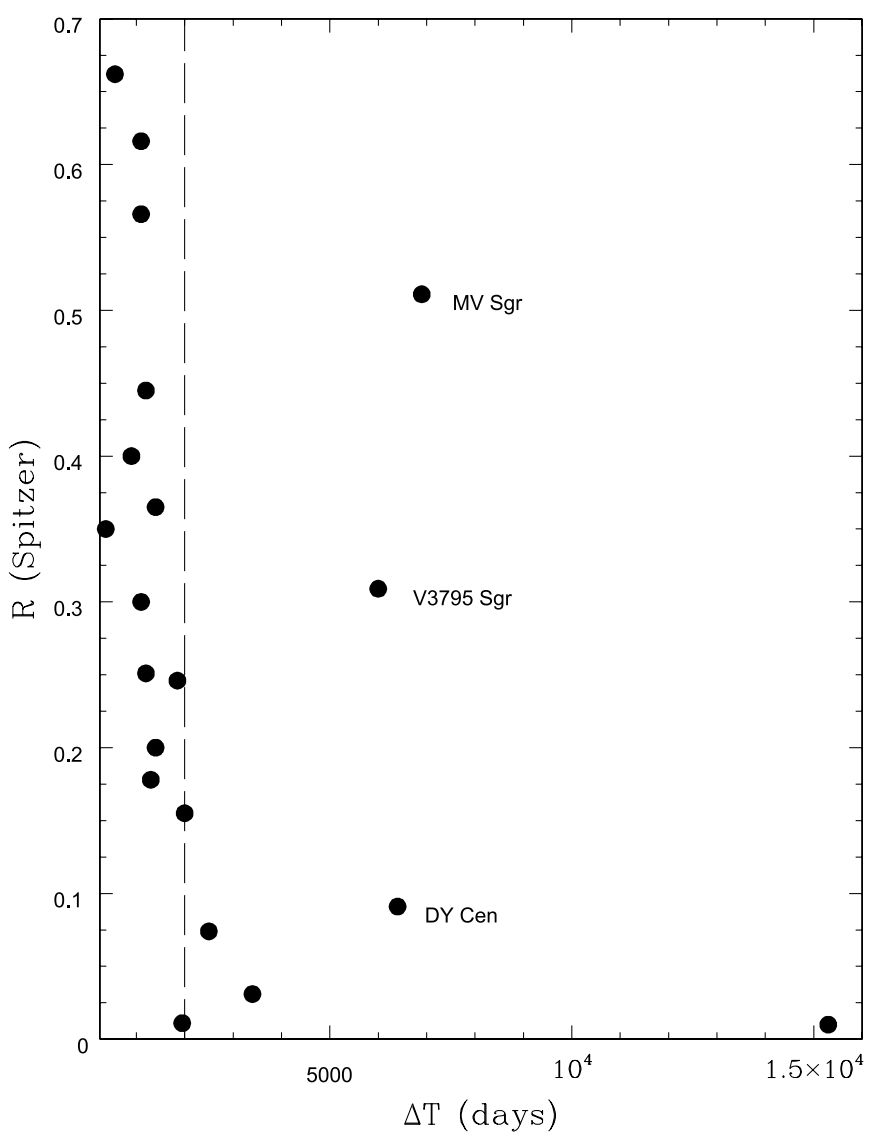

Figure 13. Spitzer covering factor $R$ versus the inter-fade period $\Delta T$. The dashed vertical line marks the point $\Delta T=2000$ days discussed in the text. Note that UV Cas with the longest $\Delta T=25,500$ days and a very low $R=0.035$ is not shown for clarity.

five years. A distinguishing mark of RY Sgr is its periodic Cepheid-like variation in optical light and the consequent variation in the infrared emission by the dust which is heated by optical light (Feast et al. 1997; Feast 1979, 1986). The amplitude of the effect at $L$ is about 0.8 mag or a factor of two. Without a correction for this pulsational variation, a comparison of IRAS and Spitzer fluxes is not immediately interpretable solely in terms of dust ejection episodes. Nevertheless, we suggest that RY Sgr behaves similarly to WX CrA in terms of dust emission.

\subsection{Covering Factors and Frequency of Declines}

There is a not unexpected correlation between the covering factor $R$ and the frequency of declines. As a measure of the latter, we use the inter-fade period $\Delta T$ (in days) determined by Jurcsik (1996). In Figure 13, we show $\Delta T$ versus $R$. Apart from the hot RCB MV Sgr, $R$ appears independent of $\Delta T$ for values of $\Delta T>2000$ days but increases steeply with decreasing $\Delta T$ below 2000 days. The mean $\Delta T$ for stars with $R<0.30$ is 5600 days from 13 stars with the subset of 5 stars with $R<0.10$ giving a mean $\Delta T=10,300$ days. For $R<0.30$, the mean is $\Delta T=1000$ days from 7 stars.

The simplest interpretation of this correlation is that the interfade period is roughly the time between ejection of puffs from all or most parts of the star and that these puffs take on the order of 2000 days to move out to distances where the dust temperature is lower than about $100 \mathrm{~K}$. Ten stars in Table 3 were not studied by Jurcsik. Of these, the data on $V$ magnitudes in the last nine years for six stars (V1783 Sgr, V1157 Sgr, V739 Sgr, ES Aql, FH Sct, and V517 Oph) are available in the ASAS-3 database and appear to be consistent with the correlation suggested by Figure 13 . With the exception of V1783 Sgr (with $\Delta T \sim 1000$ days), the other five stars are frequently in decline (at least five declines in the last nine years). The data for the other four stars (Z Umi, MACHOJ181933, DY Per, and HV 2671) are too sparse to estimate their $\Delta T$.

\subsection{On the Back of an Envelope}

As a guide to aspects of the infrared emission by dust in the circumstellar shell, the following back-of-an-envelope calculations are offered.

The equilibrium temperature of a gray dust grain $\left(T_{d}(r)\right)$ in an optically thin circumstellar environment is given by

$$
T_{d}(r)=\left(\frac{R_{*}}{2 r}\right)^{0.5} T_{*},
$$

where $R_{*}$ is the stellar radius, $r$ is the radial distance from the stellar center, and $T_{*}$ is the stellar blackbody temperature (Kwok 2007 , p. 314, Equation (10.32)). For $T_{*}=6000 \mathrm{~K}$, a representative temperature for a warm $\mathrm{RCB}$, the dust temperature is $1320 \mathrm{~K}$ at 10 stellar radii and falls to $500 \mathrm{~K}$ at 50 stellar radii. This temperature at 10 stellar radii is close to the condensation temperature of carbon soot. The temperature at 50 stellar radii is fairly typical of the warm blackbody temperature from the fit to the Spitzer spectra.

If the velocity of the dust is expressed in units of $10 \mathrm{~km} \mathrm{~s}^{-1}$ and the radius of the star in units of 100 solar radii, the time for dust to travel out from a distance $r_{i} / R_{*}$ to $r_{f} / R_{*}$ is given by

$$
t=0.22 \frac{R_{100}}{v_{10}}\left[\frac{r_{f}}{R_{*}}-\frac{r_{i}}{R_{*}}\right],
$$

where $t$ is expressed in years. For example, dust moving at $10 \mathrm{~km} \mathrm{~s}^{-1}$ will take about 10 years to move from 10 to 50 stellar radii assuming the RCB radius is about 100 solar radii, which is representative of yellow supergiants like the RCBs. A velocity of $10 \mathrm{~km} \mathrm{~s}^{-1}$ is a typical value for circumstellar gas around normal dusty AGB stars. Very blueshifted components to the $\mathrm{Na} \mathrm{D}$ lines are seen at the time of recovery from a deep minimum - say, $300 \mathrm{~km} \mathrm{~s}^{-1}$ (Rao et al. 1999). The evolution of the RCB spectra and light curves during declines is consistent with dust that forms close to the stellar atmosphere and then is accelerated to hundreds of $\mathrm{km} \mathrm{s}^{-1}$ by radiation pressure (e.g., Clayton et al. 1992; Whitney et al. 1993). There is also some evidence that the strength of the blueshifted absorption seen in He I $10830 \AA$ is inversely correlated with time since the last decline (Geballe et al. 2009; G. C. Clayton 2011, in preparation). At present, the location of this gas and its relation to the thinning puff are unknown. However, our results indicate that the dust detected at the Spitzer and IRAS wavelengths is expanding at maybe tens of $\mathrm{km} \mathrm{s}^{-1}$ but not hundreds (see below). In deep declines, the photospheric spectrum is often "washed out," an effect attributed to scattering of photospheric light off the puffs in the circumstellar environment. There is no direct way to measure the velocity of the dust because of the lack of sharp spectroscopic features that provide radial velocities. Indeed, the nearest method to measure the velocity of the dust is to study the dust-scattered photospheric spectrum of the star by moving (expanding) the dust cloud. Such a model has been shown by Herbig (1969), Kwok (1976), and in a more detailed way by Van Blerkom \& Van Blerkom (1978), where they show the 
change in profiles of stellar absorption lines by expanding dust and the redshifts expected. We have measured such redshifts of the absoprtion lines in three minima of $\mathrm{R} \mathrm{CrB}$ including the current one. Our Keck high-resolution $(30,000)$, high S/ $\mathrm{N}$ spectra obtained when the star is at $V \sim 15$ th show the scattered stellar absorption spectrum very clearly and redshifts of about $25 \mathrm{~km} \mathrm{~s}^{-1}$ relative to the mean radial velocity of the star at maximum light, suggesting dust velocities of this order. We have also broadened the normal light maximum spectrum and matched to the scattered spectrum with such velocities, suggesting also that dust is expanding at maybe tens of $\mathrm{km} \mathrm{s}^{-1}$ but not hundreds (N. K. Rao \& D. L. Lambert 2011, in preparation). Thus, if the puffs are moving radially away from the star, an expansion velocity of $10-20 \mathrm{~km} \mathrm{~s}^{-1}$ seems to be well justified. Bogdanov et al. (2010)_by using calculations where the momentum couples the gas and dust in a self-consistent procedure-estimate the characteristics of the stellar wind due to radiation pressure on the dust in two RCBs. Consistent with our suggested expansion velocity, they compute gas and dust expansion rates of 8.8 and $15.6 \mathrm{~km} \mathrm{~s}^{-1}$ for the RCBs UV Cas and SU Tau, respectively, supporting our independent estimation of the dust expansion velocity in RCBs and suggesting that the dust detected by Spitzer and IRAS may not be close to the star where dust formation seems to occur. Finally, note that with dust cloud complex motions of $10-20 \mathrm{~km} \mathrm{~s}^{-1}$ and assuming a distance of $2 \mathrm{kpc}$, the $18^{\prime}$ diameter cool (30 K, at wavelengths beyond $60 \mu \mathrm{m}$ ) dust shell seen around R CrB (Rao \& Nandy 1986; Gillett et al. 1986) would have left the star 256,000 years ago. Thus, this cool dust component may be the remnant of the stage when R CrB was a red giant for the first time (Rao \& Nandy 1986).

Thus, an RCB experiencing infrequent ejections of dust would be expected to show significant variations in infrared flux on timescales of a decade or two, i.e., differences should be seen in comparing IRAS and Spitzer fluxes for those stars which rarely emit puffs. Conversely, stars ejecting puffs at a rate much shorter than this timescale should show a quasi-constant infrared excess where the covering factor will depend in part on the total solid angle subtended by puffs which in turn will depend on the number of ejection sites close to the stellar surface and the angular expansion of a puff as it moves away from the star.

These order-of-magnitude estimates for dust temperature and timescale may be tested using the RCBs which are extreme outliers in the histogram of the IRAS to Spitzer $12 \mu \mathrm{m}$ flux ratios. These are stars for which one might expect a single puff to be present at a given time and, hence, the same puff may have been observed by IRAS and Spitzer. Two tests are offered.

First, Equations (1) and (2) may be combined to express the timescale $t$ in terms of the blackbody temperatures from the IRAS and the Spitzer fluxes. Assuming $r_{100} / v_{10}=1$ and taking the temperatures from Table 3, we predict a timescale of 13, 20, and 28 years for UV Cas, Y Mus, and RT Nor, respectively, the three stars with the most extreme decrease in $12 \mu \mathrm{m}$ flux from IRAS to Spitzer. These estimates are very similar to the time interval of about 25 years between the IRAS and Spitzer observations. For the other two outliers, both with less extreme values of the ratio of IRAS to Spitzer fluxes, the estimated timescale is much less than the 25 year time interval but this may be due to their higher frequency of puff ejection and, hence, the presence in the circumstellar shell of more than a single puff.

The second test uses the derived covering factors and the change in these factors between the IRAS and Spitzer observations. On the assumption that the physical size of the puff is

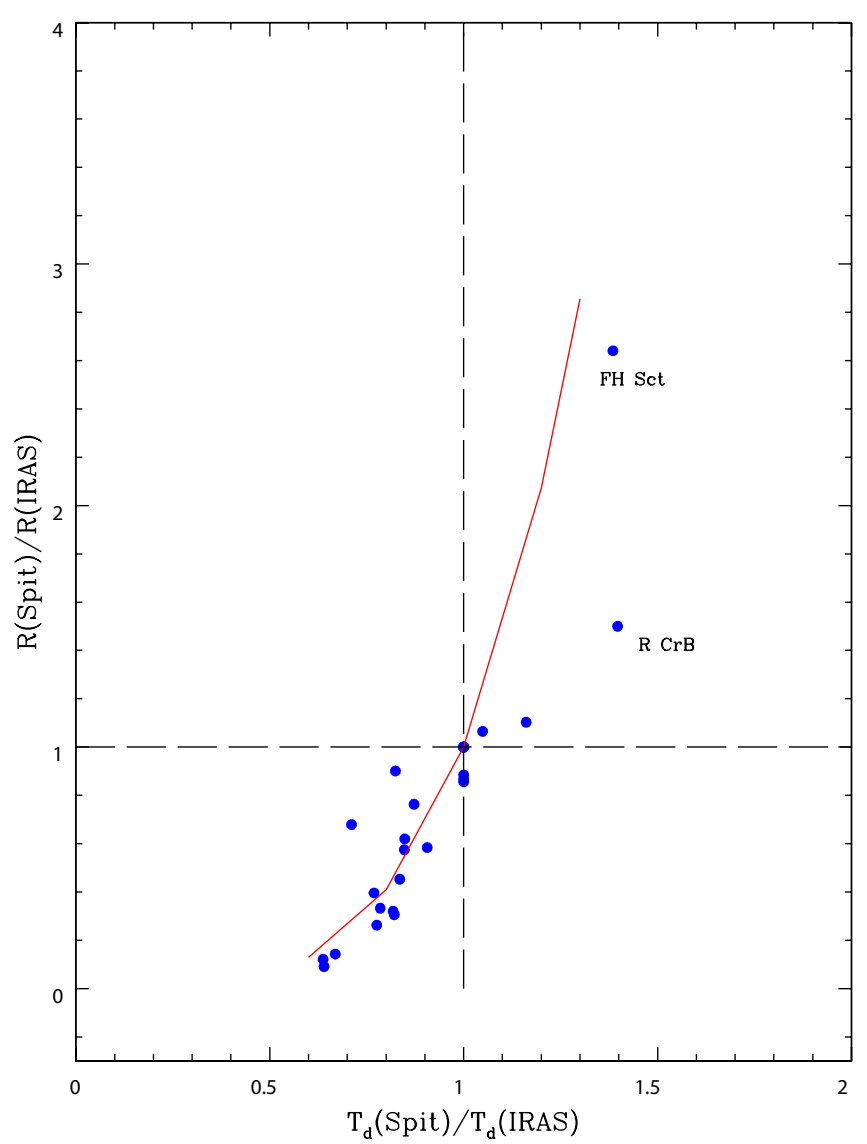

Figure 14. Ratio of the Spitzer to IRAS blackbody dust temperatures versus the ratio of the Spitzer to IRAS covering factors. The solid line is the simple prediction discussed in the text.

not changing, the covering factor $R$ and dust temperature $T_{d}$ are related as $R \propto T_{d} 4$. For the three most extreme outliers in the $I R A S$ to Spitzer flux ratio distribution, the ratio of $R$ factors and the ratio of $T_{d} 4$ are in fair accord: the $R$-ratios and $T_{d} 4$-ratios are 11 and 6, 8 and 5, and 8 and 6 for UV Cas, Y Mus, and RT Nor, respectively. Again, the indication is that a single puff present at the time of the IRAS observations remained unaccompanied at the time of the Spitzer observations but had been driven to a larger radial distance.

This test may be applied to the entire sample including outliers. In Figure 14, the ratio of the Spitzer to IRAS blackbody temperatures is plotted versus the ratio of the covering factors. Given their characteristic optical variability, it is remarkable that the RCB stars form a rather well-defined trend in this figure. On the assumption, as above, that the physical size of a puff does not change as it moves away from its star, the $R \propto T_{d} 4$ may be applied to the sample and provides the evolutionary track shown in Figure 14. Stars near the point corresponding to equal ratios in $T_{d}$ and in $R$ are identified as stars whose circumstellar environment was essentially unchanged between the IRAS and Spitzer observations. The extremity of the trend is set by the three extreme outliers (UV Cas, Y Mus, and RT Nor) for which we have suggested the principal puff present at the time of the IRAS observations evolved away from the star to greater distances and, therefore, a lower covering factor and a lower temperature. An interpretation of the fact that many stars connect the normalization point and the location of the extreme outliers is that for these stars too the principal puffs present for IRAS remained the principal puffs for Spitzer but 
then at a greater distance, Of course, this interpretation has the novel, if uncomfortable conclusion, that the majority of our collection of RCBs is now experiencing a decline in their propensity to eject puffs of soot. Since the trend is anchored by three extreme outliers, it is challenging to identify systematic errors in either the IRAS or Spitzer data that might account for this uncomfortable conclusion.

A key question about the formation and ejection of the dusty puffs is whether there is a preferred location for their formation with respect to the star. (Their ejection path is presumed to be radially outward above the point of formation.) We consider a few limiting cases.

There is a preferred location for puff formation. One imagines, for example, such possibilities as active regions at one or both rotational poles, along a latitudinal belt, or a long-lived active region (e.g., Wdowiak 1975; Soker \& Clayton 1999). The covering factor will likely be small for ejection from a single active region but larger for a fairly uniformly active latitudinal belt.

In this circumstance, optical declines will be less frequent than the time between ejection of puffs unless the preferred direction intersects the line of sight to the star. In the extreme case, the RCB will show an infrared excess but very rarely or never an optical decline.

If the time between fresh puffs is long, the infrared excess will move from near- to far-infrared wavelengths before ejection of a fresh puff, i.e., there will be large variations in flux at $L$ and at Spitzer/IRAS wavelengths.

At the other extreme when puffs are ejected frequently, the star will show smaller variations in infrared excess with a broader distribution in infrared wavelengths.

The outliers such as UV Cas, Y Mus, and RT Nor are candidates for puff ejection occurring-currently-from an active region which ejects puffs off the line of sight. This region may or may not represent a preferred location in the long term. Rao \& Raveendran (1993) have suggested a preferred plane for dust around V854 Cen from polarimetry during two deep minima. Clayton et al. (1997), from their spectro-polarimetric observations of $\mathrm{R} \mathrm{CrB}$ in a deep minimum, suggested a dust disk or torus that obscures the star with diffuse dust above the poles. On the other hand, resolved dust shells around RCBs at visible and infrared wavelengths suggest different shapes ranging from spherical symmetry to slightly elliptical (Gillet et al. 1986; Walker 1986; Clayton et al. 1999; Bright et al. 2011). Thus, preferred locations are a possibility in some cases and under some circumstances.

Puff ejection occurs from regions distributed isotropically over the stellar surface. If the number of active regions is small and ejection of puffs infrequent, there will be large variations in the infrared excess and long intervals between optical declines with possibly a low covering factor. This state of affairs is generally equivalent to that expected from a preferred location for puff ejection lying off the line of sight. Again, the outliers such as UV Cas and friends fall in this category.

At the other extreme, the active regions may be many and puff ejection very frequent, the infrared variations will be small, the optical decline rate will be high, and the covering factor high. These circumstances describe well a majority of the RCBs studied here.

These conclusions drawn from Spitzer and IRAS flux similarities and differences echo those given earlier by Feast et al. (1997) from their two decades of JHKL photometry of about a dozen RCBs. The $J$ magnitude is set by the stellar flux and is sensitive to optical declines. The $L$ magnitude monitors the dust emission, especially the warm and presumably freshly formed dust; Feast (1997) suggests that there is no evidence for dust warmer than about $1500 \mathrm{~K}$. Not surprisingly, the $L$ magnitude of a given RCB is variable with an amplitude of up to three magnitudes with larger $L$ variations associated with the longer timescales. There is a very approximate tendency for the larger $L$ variations to come from stars with longer inter-fade periods, a correlation consistent with our observation that the larger covering factors are paired with the shorter inter-fade periods. Significantly, Feast et al. (1997) conclude that "models involving fixed geometry for the ejection of dust from the star appear to be ruled out and the data support the random dust-puff model." This remark derived from the dust as measured by the $L$ magnitudes refers pretty directly to the formation of fresh dust quite close to a star.

\section{DUST AROUND RCBs IN DIFFERENT ENVIRONMENTS}

How different or similar is the dusty circumstellar shell around RCB stars in different environments (e.g., at different metallicities) such as the solar neighborhood and the LMC?

The properties of dust around RCBs in the LMC have been explored by Tisserand et al. (2009) using the photometry from Spitzer Infrared Array Camera (IRAC) and Multiband Imaging Photometer (MIPS) bands. In various diagnostic diagrams such as the [24.0] absolute magnitude versus the [8.0]-[24.0] color (as estimated from Spitzer mid-IR bands and the LMC's distance modulus), the LMC cool (i.e., DY Per-like), warm, and hot RCBs are distinguishable. The DY Per-like dust shells have the bluest colors and lowest absolute magnitudes with the hotter stars trending to higher absolute magnitudes and redder colors.

For the RCBs in our sample, we obtained monochromatic fluxes at 8.0 and $24.0 \mu \mathrm{m}$ from the reddening-corrected Spitzer spectra. We obtained magnitudes in the system of IRAC [8.0] and MIPS [24.0] $\mu \mathrm{m}$ by using the appropriate flux calibrations (Engelbracht et al. 2007) and IRAC manuals. It is to be noted that our [8.0] and [24.0] magnitudes are not band-averaged as is the standard photometry used by Tisserand et al. (2009). However, systematic effects between our magnitudes and those from Tisserand et al. (2009) are thought to be small. For the few stars where our Spitzer spectrum does not extend to $8 \mu \mathrm{m}$, we used the blackbody fits that characterize the observed spectrum (Section 3) to estimate the flux at $8 \mu \mathrm{m}$. In addition, for stars like V854 Cen and DY Cen where the 6-10 $\mu \mathrm{m}$ emission features dominate the spectra, we used the emission-free continuum flux at the appropriate wavelengths.

For our comparison with the LMC's RCBs, we must estimate the distances to the local RCBs. We have assumed $M_{\mathrm{bol}}=$ $-5.0 \sim M_{V}$ for most of the stars, with the exception of the cooler RCBs (see below). Individual distances are estimated from the reddening-corrected $V$ magnitudes from which $M_{\text {bol }}$ at 8 and $24 \mu \mathrm{m}$ were obtained. For DY Per, the distance of $2.7 \mathrm{kpc}$ as estimated by Začs et al (2007) has been adopted. Tisserand et al. (2009) show that the $M_{V}$ of RCBs in the LMC change from -5 to -3.4 as a function of the $V-I$ color, particularly for $V$ - $I$ between 0.6 and 1.5 (their Figure 3), suggesting that cooler RCBs have lower $M_{V} \mathrm{~s}$. We have used the $V-I$ color of the cooler RCBs in our sample to obtain $M_{V}$ estimates except for $\mathrm{U}$ Aqr and MACHOJ181933. These estimates are used in determining the distances to the individual stars and these distances are in turn used in estimating the absolute magnitude at $24 \mu \mathrm{m}$. U Aqr 


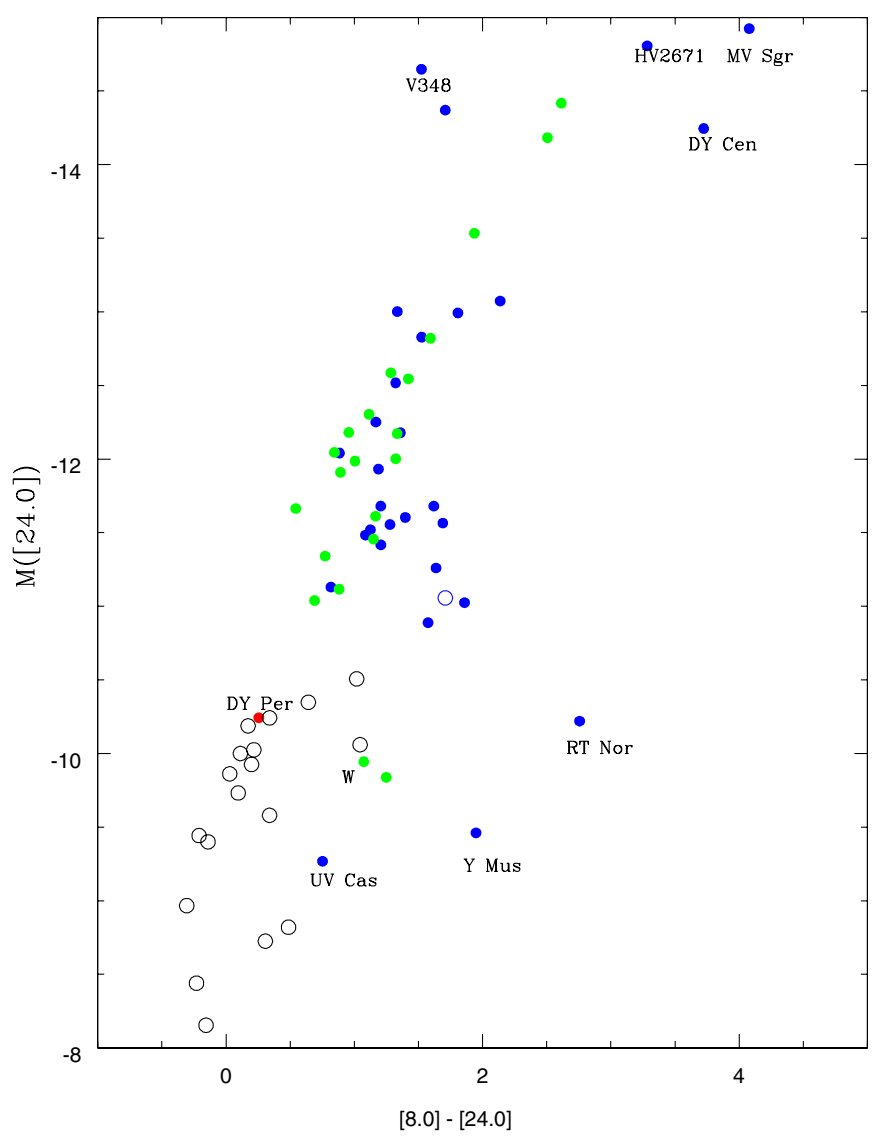

Figure 15. [24.0] $\mu \mathrm{m}$ absolute magnitude vs. the [8.0]-[24.0] color index for our sample of Galactic RCBs (blue dots) in comparison with LMC RCBs (green dots) and LMC DY Per-like stars (black open circles). Hot RCBs (V348 Sgr, DY Cen, MV Sgr, and HV 2671) are located toward the [24.0] $\mu \mathrm{m}$ highluminosity end and are labeled. The three RCB stars (UV Cas, Y Mus, and RT Nor) showing remarkably low [24.0] $\mu \mathrm{m}$ magnitudes are also labeled. Note that DY Per itself and W Men are also marked with a red dot and the letter "W," respectively.

is a halo star and the $M_{V}$ of -5 seems to be justified (Cottrell \& Lawson 1998). MACHOJ181933 displays the largest $V-I$ color in our sample and we have assumed $M_{V}=-5.0$ for this star. This is because Tisserand et al. (2009) show that there is not a unique relation between $M_{V}$ and $V-I$ color for $V-I$ colors greater than 1.5.

Figure 15 shows our sample of Galactic RCBs in the [24.0] absolute magnitude versus the [8.0]-[24.0] color index plane, along with the LMC RCBs studied by Tisserand et al. (2009). The latter data were taken from Table 6 of Tisserand et al. (2009), in which a distance modulus of 18.5 was used for the LMC objects.

The only DY Per-like object in our sample is DY Per itself, which merges with the LMC DY Per-like objects, suggesting that the distance estimate of DY Per is not too far off. The other LMC RCBs and Galactic RCBs merge in this plot over the total range, suggesting that the dusty circumstellar shells are of similar nature. The hot RCBs are found at the highest [24.0] $\mu \mathrm{m}$ luminosities while the Galactic Center cool RCB MACHOJ181933 is found at $M([24.0])=-14.37$. However, if we assume a distance modulus of 14.4 for the Galactic Center, then the [24.0] $\mu \mathrm{m}$ luminosity is reduced to -11.06 (see Figure 15), which is consistent with the other RCBs in our sample and implies that $M_{V}$ is not -5.0 for MACHOJ181933. Tisserand et al. (2009) found that the $M_{V}$ of RCBs extend from
-5.2 to -3.4 or even -2.6 . The RCBs UV Cas, Y Mus, and RT Nor along with W Men (denoted as W in Figure 15) lie far below the [24.0] $\mu \mathrm{m}$ magnitude of other RCBs although they have similar [8.0]-[24.0] colors. This suggests that these stars have lower than average dust content. Indeed, UV CAs, Y Mus, and RT Nor are just the three outliers (showing the highest IRAS/Spitzer flux ratios; see Section 4) displaying the lowest (0.01-0.035) covering factors in our sample, being very poor producers of dust. In short, we conclude that RCBs in the LMC and the Galaxy have similar dusty circumstellar shells.

\section{CONCLUSIONS}

Our almost-complete sample of Spitzer/IRS spectra of RCBs has been combined with multi-color photometry of an RCB at maximum light to provide an SED from, in general, the $U$ or $V$ band to $37 \mu \mathrm{m}$. Each SED has been fitted with a blackbody to represent the stellar flux and one, two, or three blackbodies to represent the emission from the circumstellar dust. A typical RCB emits about $30 \%$ of the stellar flux in the infrared. Although not discussed in this paper, emission features superimposed on the combination of dust blackbodies are (with a couple of exceptions) limited to emission from 6 to $10 \mu \mathrm{m}$. The exceptions discussed by García-Hernández et al. (2011) are DY Cen and V854 Cen where emission is from PAHs and $\mathrm{C}_{60}$ molecules (see also García-Hernández et al. 2010b).

For the majority of the RCBs, there is fair agreement between the Spitzer spectrum and the $12 \mu \mathrm{m}$ and $25 \mu \mathrm{m}$ fluxes from $I R A S$ from about three decades previously. There are five exceptions where the IRAS fluxes are between three to five times those recorded by Spitzer. Oddly, these outliers do not have counterparts for which the IRAS fluxes are lower than the Spitzer values.

Our results are consistent with the proposal that clouds of carbon soot form in puffs above the surface of an RCB. There is evidence that puffs are formed randomly and without a preferred direction. For some stars, and especially for the above five outliers, a single puff may dominate the infrared emission. Our sample of Galactic RCBs and those in the LMC share the same properties of their dusty circumstellar shells, as evidenced by their total $24 \mu \mathrm{m}$ luminosity and [8.0]-[24.0] color index.

Although an important next step is to attempt to model the radiative transfer in the dusty circumstellar environment for our sample of RCB stars, the value of long-term monitoring through infrared photometry and spectroscopy should not be underestimated.

We thank the referee G. C. Clayton for suggestions that helped to improve the paper. We thank Michael Feast, Patricia Whitelock, and Fred van Wyk at the SAAO for observing stars at the time of our Spitzer observations. We also thank N.M. Ashok of PRL, Ahmedabad for observing several RCB stars at the Gurushikhar Observatory. We thank John Lacy and Amanda Bayless for discussions about modeling the dusty environment of RCB stars. D.A.G.H. acknowledges support for this work provided by the Spanish Ministry of Science and Innovation (MICINN) under the 2008 Juan de la Cierva Programme and under grant AYA-2007-64748. N.K.R. would like to thank A. V. Raveendran for his help. D. L. L. acknowledges support for this work provided by NASA through an award for program GO 50212 issued by JPL/Caltech and the Robert A. Welch Foundation of Houston, Texas through grant F-634. Extensive use has been made of the AAVSO's database of observations of RCB stars; we are most grateful to the many observers who have 
and continue to contribute to this record of the stars' variability. This work is based on observations made with the Spitzer Space Telescope, which is operated by the Jet Propulsion Laboratory, California Institute of Technology, under NASA contract 1407. Part of this work is based on observations made with the IAC-80 telescope under the Spanish Instituto de Astrofísica de Canarias Service Time. The IAC- 80 is operated by the Instituto de Astrofísica de Canarias in the Observatorio del Teide. Facilities: Spitzer, OT:0.8m, SAAO:0.75m

\section{REFERENCES}

Alcock, C., Allsman, R. A., Alves, D. R., et al. 2001, ApJ, 554, 298 Alksnis, A., Larionov, V. M., Smirnova, O., et al. 2009, Balt Astron., 18, 53 Asplund, M., Gustafsson, B., Kiselman, D., \& Eriksson, K. 1997, A\&A, 318, 521

Asplund, M., Gustafsson, B., Lambert, D. L., \& Rao, N. K. 2000, A\&A, 353, 287

Asplund, M., Gustafsson, B., Rao, N. K., \& Lambert, D. L. 1998, A\&A, 332, 651

Beichman, C. A., Neugebauer, G., Habing, H. J., Clegg, P. E., \& Chester, T. J. 1988, Infrared Astronomical Satellite (IRAS) Catalogs and Atlases, Vol. 1: Explanatory Supplement (Washington, DC: NASA)

Benson, P. J., Clayton, G. C., Garnavich, P., \& Szkody, P. 1994, AJ, 108, 247

Bergeat, J., Knapik, A., \& Rutily, B. D. 1999, A\&A, 342, 773

Bogdanov, M. B., Taranov, O. G., \& Shenavrin, V. I. 2010, Astron. Rep., 54, 620

Bond, H. E., Luck, R. E., \& Newman, M. J. 1979, ApJ, 233, 205

Bright, S. N., Chesneau, O., Clayton, G. C., et al. 2011, MNRAS, 414, 1195

Cardelli, J. A., Clayton, G. C., \& Mathis, J. S. 1989, ApJ, 345, 245

Carter, B. S. 1990, MNRAS, 242, 1

Chiar, J. E., \& Tielens, A. G. G. M. 2006, ApJ, 637, 774

Clayton, G. C. 1996, PASP, 108, 225

Clayton, G. C., Bjorkman, K. S., Nordsieck, K. H., Zellner, E. B., \& SchulteLadbeck, R. E. 1997, ApJ, 476, 870

Clayton, G. C., De Marco, O., Whitney, B. A., et al. 2011, AJ, in press (arXiv:1106.0563)

Clayton, G. C., Geballe, T. R., Herwig, F., Fryer, C., \& Asplund, M. 2007, ApJ, 662,1220

Clayton, G. C., Hammond, D., Lawless, J., et al. 2002, PASP, 114, 846

Clayton, G. C., Herwig, F., Geballe, T. R., et al. 2005, ApJ, 623, L141

Clayton, G. C., Kelly, D. M., Lacy, J. H., et al. 1995, AJ, 109, 2096

Clayton, G. C., Kerber, F., Gordon, K. D., et al. 1999, ApJ, 517, L143

Clayton, G. C., Whitney, B. A., Stanford, S. A., \& Drilling, J. S. 1992, ApJ, 397,652

Colangeli, L., Mennella, V., Palumbo, P., Rotundi, A., \& Bussoletti, E. 1995, A\&AS, 113,561

Cottrell, P. L., \& Lambert, D. L. 1982, Observatory, 102, 149

Cottrell, P. L., \& Lawson, W. A. 1998, PASA, 15, 179

Cox, N. L. J., García-Hernández, D. A., García-Lario, P., \& Manchado, A. 2011, AJ, 141, 111

Crause, L. A., Lawson, W. A., \& Henden, A. A. 2007, MNRAS, 375, 301

Cutri, R. M., Skrutskie, M. F., van Dyk, S., et al. 2003, The 2MASS All-Sky Point Source Catalog, Vizier Online Catalog, 2246, 0

Danziger, I. J. 1965, MNRAS, 130, 199

de Laverny, P., \& Mékarnia, D. 2004, A\&A, 428, L13

De Marco, O., Clayton, G. C., Herwig, F., et al. 2002, AJ, 123, 3387

Engelbracht, C. W., Blaylock, M., Su, K. Y. L., et al. 2007, PASP, 119, 994

Feast, M. W. 1979, in IAU Colloq. 46, Changing Trends in Variable Star Research, ed. F. M. Bateson, J. Smak, \& I. M. Urch (Hamilton: Univ. Waikato), 246

Feast, M. W. 1986, in IAU Colloq. 87, Hydrogen Deficient Stars and Related Objects, ed. K. Hunger, D. Schönberner, \& N. K. Rao (Dordrecht: Reidel), 151

Feast, M. W. 1996, in ASP Conf. Ser. 96, Hydrogen Deficient Stars, ed. C. S. Jeffery \& U. Heber (San Francisco, CA: ASP), 3

Feast, M. W. 1997, MNRAS, 285, 339

Feast, M. W., Carter, B. S., Roberts, G., Marang, F., \& Catchpole, R. W. 1997, MNRAS, 285, 317

Feast, M. W., \& Glass, I. S. 1973, MNRAS, 161, 293

Fernie, J. D., Sherwood, V., \& DuPuy, D. L. 1972, ApJ, 172, 383

Fitzgerald, M. P. 1968, AJ, 73, 683

Forrest, W. J., Gillett, F. C., \& Stein, W. A. 1972, ApJ, 178, L17

García-Hernández, D. A., Hinkle, K. H., Lambert, D. L., \& Eriksson, K. 2009a, ApJ, 696, 1733
García-Hernández, D. A., Lambert, D. L., Rao, N. K., Hinkle, K. H., \& Eriksson, K. 2010, ApJ, 714, 144

García-Hernández, D. A., Manchado, A., García-Lario, P., et al. 2010b, ApJ, 724, L39

García-Hernández, D. A., Perea-Calderón, J. V., Bobrowsky, M., \& GarcíaLario, P. 2007, ApJ, 666, L33

García-Hernández, D. A., Perea-Calderón, J. V., Engels, D., \& GarcíaLario, P. 2009b, in Asymmetrical Planetary Nebulae IV, ed. R. L. M. Corradi, A. Manchado, \& N. Soker (IAC electronic publication: www.iac.es/proyecto/apn4/pages/proceedings.php), 325

García-Hernández, D. A., Rao, N. K., \& Lambert, D. L. 2011, ApJ, 729, 126

Gaustad, J. E., Stein, W. A., Forrest, W. J., \& Pipher, J. L. 1988, PASP, 100, 388

Geballe, T. R., Rao, N. K., \& Clayton, G. C. 2009, ApJ, 698, 735

Gillett, F. C., Backman, D. E., Beichman, C., \& Neugebauer, G. 1986, ApJ, 310, 842

Glass, I. S. 1978, MNRAS, 185, 23

Goldsmith, M. J., Evans, A., Albinson, J. S., \& Bode, M. F. 1990, MNRAS, 245,119

Goswami, A., Rao, N. K., \& Lambert, D. L. 1997, PASP, 109, 796

Heck, A., Houziaux, L., Manfroid, J., Jones, D. H. P., \& Andrews, P. J. 1985, A\&AS, 61, 375

Herbig, G. H. 1969, Mém. Soc. R. Sci. Liege, 19, 13

Higdon, S. J. U., Devost, D., Higdon, J. L., et al. 2004, PASP, 116, 975

Hoffleit, D. 1959, AJ, 64, 241

Houck, J. R., Roellig, T. L., van Cleve, J., et al. 2004, ApJS, 154, 18

Jeffery, C. S. 1995, A\&A, 297, 779

Jeffery, C. S., \& Heber, U. 1993, A\&A, 270, 167

Jeffery, C. S., Heber, U., Hill, P. W., \& Pollacco, D. 1988, MNRAS, 231, 175

Jeffery, C. S., Karakas, A. I., \& Saio, H. 2011, MNRAS, 414, 3599

Jurcsik, J. 1996, Acta Astron., 46, 325

Kilkenny, D., Coulson, I. M., Laing, J. D., Spencer Jones, J., \& Engelbrecht, C. 1985, SAAO Circ., 9, 87

Kilkenny, D., Lloyd Evans, T., Bateson, F. M., Jones, A. F., \& Lawson, W. A. 1992, Observatory, 112, 158

Kilkenny, D., \& Whittet, D. C. B. 1984, MNRAS, 208, 25

Kipper, T., \& Klochkova, V. G. 2006, Balt. Astron., 15, 531

Kwok, S. 1976, J. R. Astron. Soc. Can., 70, 49

Kwok, S. 2007, Physics and Chemistry of the Interstellar Medium (Sausalito: Univ. Science Books)

Lambert, D. L., \& Rao, N. K. 1994, J. Astrophys. Astron., 15, 47

Lambert, D. L., Rao, N. K., Pandey, G., \& Ivans, I. I. 2001, ApJ, 555, 925

Lawson, W. A., \& Cottrell, P. L. 1997, MNRAS, 285, 266

Lawson, W. A., Cottrell, P. L., Kilmartin, P. M., \& Gilmore, A. C. 1990, MNRAS, 247,91

Lawson, W. A., Maldoni, M. M., Clayton, G. C., et al. 1999, AJ, 117, 3007

Leão, I. C., de Laverny, P., Chesneau, O., Mékarnia, D., \& de Medeiros, J. R. 2007, A\&A, 466, L1

Lloyd Evans, T., Kilkenny, D., \& van Wyk, F. 1991, Observatory, 111, 244

Marang, F., Kilkenny, D., Menzies, J. W., \& Spencer Jones, J. H. 1990, SAAO Circ., 14, 1

Pandey, G., \& Lambert, D. L. 2011, ApJ, 727, 122

Pollacco, D. L., \& Hill, P. W. 1991, MNRAS, 248, 572

Pollacco, D. L., Hill, P. W., Houziaux, L., \& Manfroid, J. 1991, MNRAS, 248, 1

Pollacco, D. L., Hill, P. W., \& Tadhunter, C. N. 1990, MNRAS, 245, 204

Pugach, A. E. 1977, IBVS, 1277, 1

Rao, N. K. 1980, Observatory, 100, 164

Rao, N. K. 1995, Bull. Astron. Soc. India, 23, 351

Rao, N. K., Ashok, N. M., \& Kulkarni, P. V. 1980, J. Astrophys. Astron., 1, 71

Rao, N. K., Giridhar, S., \& Lambert, D. L. 1993, A\&A, 280, 201

Rao, N. K., \& Lambert, D. L. 1993, PASP, 105, 574

Rao, N. K., \& Lambert, D. L. 2000, MNRAS, 313, L33

Rao, N. K., \& Lambert, D. L. 2008, MNRAS, 384, 477

Rao, N. K., \& Lambert, D. L. 2010, in Recent Advances in Spectroscopy: Theoretical, Astrophysical and Experimental Perspectives, ed. R. J. Chaudhuri, M. V. Mekkaden, A. V. Raveendran, \& A. S. Narayanan (Berlin: Springer), 177

Rao, N. K., Lambert, D. L., Adams, M. T., et al. 1999, MNRAS, 310, 717

Rao, N. K., Lambert, D. L., \& Shetrone, M. D. 2006, MNRAS, 370, 941

Rao, N. K., \& Nandy, K. 1986, MNRAS, 222, 357

Rao, N. K., \& Raveendran, A. V. 1993, A\&A, 274, 330

Rosenbush, A. E. 1995, Astron. Nachr., 316, 213

Scott, A. D., Duley, W. W., \& Jahani, H. R. 1997, ApJ, 490, L175

Siedel, Th. 1957, Mitt. Veränderliche Sterne, Nr. 244

Soker, N., \& Clayton, G. C. 1999, MNRAS, 307, 993

Soszyński, I., Udalski, A., Szymański, M. K., et al. 2009, Acta Astron., 59, 335

Stein, W. A., Gaustad, J., Gillett, F. C., \& Knacke, R. F. 1969, ApJ, 155, L3 
Strecker, D. W. 1975, AJ, 80, 451

Tenenbaum, E. D., Clayton, G. C., Asplund, M., et al. 2005, AJ, 130, 256

Tisserand, P., Wood, P. R., Marquette, J. B., et al. 2009, A\&A, 501, 985

Tokunaga, A. T. 2000, in Allen's Astrophysical Quantities, ed. A. N. Cox (4th ed.; New York: Springer)

van Blerkom, J., \& van Blerkom, D. 1978, ApJ, 225, 482

Vanture, A. D., Zucker, D., \& Wallerstein, G. 1999, ApJ, 514, 932

Walker, H. J. 1986, in IAU Coll. 87, Hydrogen Deficient Stars \& Related Objects, ed. K. Hunger, D. Schönberner, \& N. K. Rao (Dordrecht: Reidel), 407

Wdowiak, T. J. 1975, ApJ, 198, L139

Werner, M. W., Roellig, T. L., Low, F. J., et al. 2004, ApJS, 154, 1
Whitney, B. A., Balm, S. P., \& Clayton, G. C. 1993, in ASP Conf. Ser. 45 Luminous High-Latitude Stars, ed. D. S. Dimitar (San Francisco, CA: ASP), 115

Woitke, P., Goeres, A., \& Sedlmayr, E. 1996, A\&A, 313, 217

Yakovina, L. A., Pugach, A. F., \& Pavlenko, Ya. V. 2009, Astron. Rep., 53, 187

Začs, L., Mondal, S., Chen, W. P., et al. 2007, A\&A, 472, 247

Zaniewski, A., Clayton, G. C., Welch, D. L., et al. 2005, AJ, 130 2293

Zavatti, F. 1975, IBVS, 1027, 1

Zhilyaev, B. E., Orlov, M. Ya., Pugach, A. F., Rodriges, M. G., \& Totochava, A. G. 1978, R Coronae Borealis-type Stars (Naukova dumka: Kiev) 